\title{
Révolution et évolution Les marchés du crédit notarié en France, 1780-1840
}

\author{
Philip T. Hoffman, Gilles Postel-Vinay \\ et Jean-Laurent Rosenthal
}

Pensons aux représentations que véhicule l'opposition classique entre histoire locale et histoire nationale. Dans le cas des régions françaises, par exemple, coexistent deux traditions. L'une, plus culturelle, privilégie les us et coutumes locaux. Tout en mettant l'accent sur la résistance persistante des cultures locales à l'assimilation dans une culture nationale, elle s'attriste (ou se félicite) de voir cette continuité contrebattue par une (inévitable) modernisation. L'autre, plus politique, fait remonter à la monarchie absolue et plus encore à la Révolution la rupture qui s'opère entre l'espace compartimenté des provinces et celui, un et indivisible, de l'État-nation.

Mutatis mutandis, des hypothèses voisines peuvent fournir un point de départ pour une recherche sur l'histoire des marchés que nous aborderons ici par le cas du crédit. Ainsi, l'historiographie l'a souvent noté, le développement de ces marchés paraît avoir été tributaire de la présence, dans un espace donné, de tel ou tel ensemble de croyances. En particulier, une des institutions importantes des marchés du crédit est, on le sait, la réputation de leurs intermédiaires puisque celle-ci fait naître ou, au moins, conforte la confiance que les prêteurs ont, ou n'ont pas, pour investir. Or la réputation dépend en partie d'une culture de marché que l'on considère souvent comme l'acquis durable d'une histoire locale. On rappellera de même qu'en matière de crédit chaque province avait ses contraintes légales avant 1789 et que l'histoire propre de chacune y avait encouragé des pratiques 
spécifiques. Or, il est courant d'admettre que ces pratiques ont eu une grande permanence au point de fixer des cultures locales en matière de crédit. Et si le fonctionnement du crédit dans un endroit est affecté par des phénomènes de cet ordre, on doit s'attendre à ce que ceux-ci aient imposé de strictes limites à tout projet unificateur; au projet jacobin par exemple.

À l'inverse, on sait que le crédit dépend aussi d'institutions qui rendent les emprunteurs fiables. Il peut s'agir ou bien d'institutions formelles, c'est-à-dire de règles explicites que l'application de la loi suffit à rendre exécutoires, que ce soit des régulations publiques ou des accords privés dont l'application est garantie par l'État (par exemple, une justice efficace contribuera à rendre un emprunteur fiable); ou bien d'institutions informelles, en entendant par là des règles implicites, ou qui, si elles sont explicites, ne sont pas susceptibles d'être rendues exécutoires par la loi, comme ce peut être le cas d'un système d'information privé sur les gages, par exemple. Mais, dans tous les cas, il semble aller de soi que, avec le temps, une part croissante de ces institutions s'est moulée dans le cadre des États. Les institutions si variées de l'Ancien Régime ont ainsi été profondément transformées par la Révolution, et tout spécialement les institutions formelles puisque celles-ci ont été rendues uniformes dans toute la France. Il suffira de citer l'unification de la législation sur l'usure pour comprendre qu'à cette occasion ont été supprimées bien des différences qui tendaient à créer, ici, des marchés actifs, là, des marchés déprimés. Et, dès lors que le contexte est partout le même, ne convient-il pas d'analyser l'histoire du crédit dans le cadre agrégé d'une comptabilité nationale plutôt que de se perdre dans les méandres inutiles d'une approche centrée sur les marchés locaux?

De fait, ces points de vue opposés ont inspiré deux perceptions de l'évolution des marchés quitte à mettre ainsi face à face des institutions qu'il paraît plus légitime de considérer comme des composantes d'un même ensemble. Il est en effet curieux d'isoler, d'un côté, des institutions locales liées aux dotations singulières d'une place donnée à un moment donné et, d'un autre côté, des institutions qui débordent ces frontières étroites, soit qu'elles aient été établies comme uniformes, soit qu'elles aient dû s'uniformiser pour permettre l'intégration des marchés. Des marchés intégrés incitent pourtant aux spécialisations et rendent donc les économies locales gourmandes d'institutions spécifiques. Dans cette mesure, mieux vaut donc construire une analyse des marchés et de leurs institutions en admettant qu'un système institutionnel soit différencié à une certaine échelle et, à une autre échelle, uniforme. Mais alors, il faut pouvoir mesurer l'impact des institutions propres à chaque échelle - échelles dont d'ailleurs la liste n'est pas close et dont rien ne dit qu'elle doive se limiter à la simple opposition binaire entre le local et le national.

Par ailleurs, si l'on considère que l'intégration est généralement source de gains d'efficacité, il importe de savoir quand et comment les marchés du crédit locaux se sont fondus en un marché national. Or, comme il a fallu, pour ce faire, créer des institutions et des pratiques qui permettent de faire circuler l'information non pas à l'intérieur d'un marché mais entre plusieurs, une nouvelle question se pose qui complète et déplace les deux approches évoquées. La croissance des marchés de crédit dépend en effet de la possibilité d'élargir l'espace où circule 
l'information. Pour cela, il faut une technique qui permette de maîtriser une masse de renseignements et débouche sur la création d'un savoir non plus particulier mais largement partageable entre les intermédiaires. Le problème est loin d'aller de soi car il s'agit d'un savoir que chaque intermédiaire doit sans cesse actualiser pour lui-même en rassemblant le plus possible d'informations sur toutes sortes de risques singuliers, que ceux-ci soient liés aux individus, à l'économie locale ou à la structure des marchés. Qu'un tel savoir puisse mieux se diffuser est une condition pour que le crédit se développe, que ce soit à l'intérieur d'un marché ou entre plusieurs. Mais si l'extension d'un marché passe par la diffusion du savoir et des techniques de production de ce savoir, il n'est nullement nécessaire que cette diffusion reste au niveau local ou, au contraire, ne s'opère qu'au niveau national.

Posons que, pour une grande part, ces techniques de savoir constituent un capital social. Si l'on s'en tient au cadre étroit de la relation entre un intermédiaire de crédit et ses clients, tout est simple: le premier connaît les seconds et voilà tout. Mais, pour que le circuit s'étende et qu'existent des flux de crédit de clientèle à clientèle ou de lieu à lieu, il faut que l'information puisse circuler bien au-delà. Les techniques d'intermédiation doivent donc être telles qu'elles permettent de faire transiter et savoirs et deniers. Or, comme le rappellent les travaux de l'économie géographique ${ }^{1}$, si le fait que les institutions ont des rendements croissants entraîne la concentration des activités, cette concentration n'en a pas moins des limites puisqu'elle se heurte au prix des terrains et aux coûts de transport - soit, ici, les coûts qu'il faut engager pour transférer une information fiable d'un marché à un autre. Se pose en outre un problème connexe. On doit en effet se demander dans quelles conditions une population décide de réaliser certains investissements de biens publics. En l'occurrence, les conditions en cause peuvent être assimilées à un capital social local; la population est celle des intermédiaires financiers; et les biens publics dans lesquels cette population choisit ou non d'investir consistent en diverses manières d'accumuler, de traiter ou de distribuer l'information comme en différentes pratiques de prêt.

Comprendre comment se crée (ou se détruit) ce genre de capital social et à quelles institutions il s'adosse ne va pas cependant sans quelques préalables. Il faut d'abord se donner une période d'analyse assez longue. Il faut aussi considérer un nombre suffisant de marchés. Nous analyserons donc ici l'évolution de soixante-sept marchés locaux du crédit à partir de trois coupes annuelles en 1780, 1807 et 1840 pour suivre les effets d'un choc majeur: la Révolution française. Nous montrerons ainsi que le crédit dans la France d'alors ne s'organise ni dans le cadre de petites régions ni dans l'espace unifié qu'ambitionne de construire la République nouvelle mais plutôt en deux grands ensembles - un " hémisphère Nord " et un " hémisphère Sud » en quelque sorte - au sein desquels les pratiques du crédit sont différentes et évoluent séparément. En même temps, ces deux ensembles, loin d'être homogènes, sont hiérarchisés entre villes et campagnes. De là quatre systèmes qui se repèrent 
aussi bien si l'on observe les instruments de crédit, les intermédiaires ou les circuits de formation que ces derniers se donnent. Pour expliquer ces quatre systèmes, il faut accepter que les institutions formelles et informelles se déploient dans l'espace d'une façon qui dépend de l'activité des marchés mais aussi de l'inégale répartition de la richesse.

Nous délimiterons d'abord le champ de cette recherche en revenant sur la question des institutions et de l'information. Ensuite, en examinant l'évolution des marchés étudiés, nous mettrons en évidence la très forte - et surprenante décentralisation du crédit notarié qu'entraîne la Révolution. Puis, pour expliquer la persistance de cette décentralisation, nous soumettrons à une analyse statistique l'effondrement puis la reprise de l'activité de crédit sur tous ces marchés. Enfin, nous examinerons les quatre systèmes qui se mettent en place après la Révolution.

\section{Une crise, quatre scénarios ; trois coupes, $\mathrm{N}$ marchés}

Des travaux classiques se sont donné pour objectif de reconstruire la logique interne des institutions financières d'une ville ou d'une région et les raisons de leur spécificité à partir d'un cadre local. De ce point de vue, le développement des institutions de crédit s'explique par l'existence de traits propres à l'économie ou à l'histoire d'un espace particulier, que ce soit la demande locale de crédit, les formes de la distribution ou de la transmission des biens, l'existence éventuelle d'une culture particulière concernant la garantie que la parenté apporte aux jeunes qui empruntent, le rôle de patronage des grandes familles ou encore l'incertitude. De même, est mise en avant la place des réseaux d'individus ou de familles importantes qui animent ou exploitent les marchés du crédit en tant qu'intermédiaires, comme capitalistes ou comme rentiers ${ }^{2}$.

À l'autre extrême, il existe une histoire du développement financier construite à l'échelon des pays qui prend son sens notamment quand on s'interroge d'un point de vue comparatif sur les effets des régimes légaux ou si l'on cherche à mettre en rapport l'évolution du crédit avec les agrégats des comptabilités nationales rétrospectives ou avec les choix de politique macro-économique - la création d'une banque centrale, par exemple, ou la stabilisation de la monnaie. L'ambition est alors de répondre à de grandes questions sur les liens entre crédit et croissance ou entre réformes politiques et marchés privés. Mais, comme mesurer ces liens suppose qu'aient été élaborées les séries d'une comptabilité rétrospective, un tel projet conduit à rejeter dans la préhistoire les temps obscurs qui ont précédé l'ère de la statistique publique - et ceci, alors même que l'on ne peut pas comprendre l'importance d'une unification si l'on ne se donne pas les moyens d'analyser la situation qui la précède. Faute des données nécessaires, l'histoire nationale a donc concentré ses efforts sur la période statistique, quitte à se satisfaire d'impressions sur les époques antérieures et notamment sur l'histoire des marchés locaux. Les a priori implicites sont alors aussi 
extrêmes que ceux de l'histoire locale; simplement, ils les inversent. L'hypothèse est que les marchés locaux sont le reflet d'une moyenne nationale et de phénomènes locaux sans persistance ni portée. Sur cette base, d'ailleurs, il devient simple d'allier histoire locale et histoire nationale car l'une finit quand l'autre commence.

Simple et trop simple. Il paraît donc important de mobiliser d'autres cadres d'analyse pour se défaire de ces dualités. C'est ce à quoi peut aider l'économie géographique dont les apports récents montrent, précisément, que l'histoire locale ne perd pas son intérêt quand les marchés s'harmonisent. Après s'être d'abord interrogé sur les raisons qui contribuent à la concentration des activités en ville ${ }^{3}$, ce courant d'analyse qui marque l'ensemble des sciences sociales, de l'économie à la science politique, s'interroge en effet sur ce qui fait la force d'un lieu par rapport à un autre. Dans cette perspective, certains auteurs mettent en avant des phénomènes locaux, souvent d'ordre culturel ${ }^{4}$; d'autres, l'effet des dotations économiques ${ }^{5}$; d'autres encore, celui des systèmes politiques locaux ${ }^{6}$. Il reste que, par-delà leurs différences, tous cherchent à rendre compte de l'importance de la diversité des situations locales après que se sont constituées les entités nationales, c'est-à-dire en prenant ces entités comme allant de soi et parfaitement identifiées, notamment par l'information produite par la statistique publique. Or un tel choix a au moins un inconvénient. Il amène à restreindre l'analyse aux $\mathrm{XIX}^{\mathrm{e}} \mathrm{et} \mathrm{XX}^{\mathrm{e}}$ siècles, quitte à renvoyer la diversité des situations locales à la très longue durée, sans se soucier de savoir s'il s'agit bien de phénomènes stables ou si ceux-ci relèvent plutôt du moyen terme, comme le suggèrent tant d'exemples historiques?

Ici, nous entendons plutôt étudier le devenir d'un ensemble de marchés du crédit en nous donnant une période d'observation assez longue pour embrasser tout à la fois une crise d'ampleur indiscutablement nationale - d'où le choix de la Révolution française - et ses suites. Pour une telle période, construire une histoire d'emblée agrégée à l'échelle du pays reviendrait à masquer la redistribution de

3 - Harold Hotelling, «Stability in competition», Economic journal, 39, 1929, pp. $41-57$.

4 -Parmi de nombreux exemples possibles, citons, pour la Hollande, JonatHan IsRael, The Dutch republic: its rise, greatness, and fall, 1477-1806, Oxford, The Clarendon Press, 1988; ou, pour l'Angleterre, ALAN MACFARLANE, The origin of English individualism: the family, property, and social transition, Oxford, Basil Blackwell, 1978.

5 - KenNeth L. SoKoloff et DAvid Dollar, «Agricultural seasonality and the organization of manufacturing during early industrialization: the contrast between Britain and the United States ", Journal of economic history, juin 1997, pp. 288-321 ; EvSEY D. DOMAR, «The causes of slavery or serfdom: a hypothesis ", Journal of economic history, mars 1970, pp. 18-32; Stanley L. Engerman et Kenneth L. Sokoloff, « Factor endowments, institutions, and differential path of growth among New World economies", in S. HABER (éd.), How Latin America fell behind? Essays on the economic histories of Brazil and Mexico, 1800-1914, Stanford, Stanford University Press, 1997, pp. 260-304.

6 - ROBERT D. PUTNAM, «The prosperous community: social capital and public life», The American prospect, 13, 1993, pp. 35-42.

7 - Pour prendre à nouveau Robert D. Putnam en exemple, telle est bien la démarche de Making the democracy work. Civic tradition in modern Italy, Princeton, Princeton University Press, 1992. 
l'activité économique et financière entre différents lieux au cours du temps. Or il est manifeste que cette redistribution a bien eu lieu, qu'elle a eu des effets importants sur les fortunes individuelles et qu'elle a souvent, à ce titre, constitué un enjeu politique majeur - l'État intervenant soit pour l'amoindrir soit pour l'exacerber. Inversement, dans le domaine du crédit comme dans tant d'autres, la Révolution a créé de nouvelles institutions qui devaient être partout présentes et partout identiques. Mais, même si le crédit s'est développé dans telle ou telle région grâce à l'une des créations institutionnelles de la Révolution, rien n'indique que l'innovation en question se soit diffusée dans tout le pays. Interviennent là, en effet, les contraintes d'information qu'on ne peut comprendre sans tenir compte d'un ensemble d'histoires emboîtées relevant d'échelles différentes : celle des institutions locales, celle de la division spatiale de l'économie et celle du pays.

Mais, si l'histoire des marchés en général et du crédit en particulier se joue à la fois dans le temps et dans des espaces géographiques variés (locaux, régionaux, nationaux...), l'historien est, nolens volens, contraint d'établir des priorités. Quitte à admettre, au moins dans un premier temps, de limiter les réponses que l'on peut attendre d'une telle recherche, il faut faire certains choix. Ceux-ci portent sur trois dimensions essentielles de tout marché : le type d'échanges dont il traite mais aussi l'espace et la durée où il se développe.

Nous avons choisi de nous intéresser ici au crédit parce qu'il permet d'illustrer le rôle des institutions et de la durée dans la construction de la croissance. Dans ce cas, on peut s'attendre à ce que le temps soit une dimension essentielle puisque les marchés du crédit sont d'abord des marchés de la richesse passée: les prêts reposent en grande partie sur l'épargne locale qui est souvent cumulative. On sait aussi que plus les contrats durent, plus ils courent le risque d'être rompus et donc plus les marchés où ils s'échangent sont avides d'institutions susceptibles d'éviter que les affaires finissent mal. Et comme ces institutions sont également le fruit de l'histoire, les marchés de crédit sont sujets à des effets de dépendance temporelle forts. En ce sens, ces marchés sont du côté de la longue durée. Il faut néanmoins souligner que les institutions du crédit, comme celles des marchés du foncier ou du travail, ont eu à s'adapter à l'évolution de l'économie et qu'elles sont, à ce titre, tributaires d'une histoire non pas seulement locale, mais aussi nationale voire globale.

Surtout, il ne suffit pas de reconnaître l'existence de dépendances temporelles comme une caractéristique générale des marchés financiers; encore faut-il savoir quand l'histoire importe le plus. Pour cela, il semble utile de préciser les liens entre la richesse et le crédit. Imaginons en effet ce qui se passe dans un lieu dont la spécialisation économique est en pleine expansion. Le taux de profit y est élevé, la demande de capital forte et les occasions d'investissements - disons les opportunités - nombreuses. Des organismes d'intermédiation, que ce soit sous la forme bancaire ou celle du courtage notarial, ont donc intérêt à s'y développer ${ }^{8}$. Partant, on s'attend à ce que de nouveaux intermédiaires s'installent et que les

8-Voir, par exemple, NAOMI LAMOREAUX, Insider lending: banks, personal connections, and economic development in industrial New England, New York, Cambridge University Press, 1994. 
anciens modifient leurs pratiques, ce qui devrait permettre d'augmenter le volume des prêts et de l'investissement.

Des obstacles peuvent cependant surgir. L'expansion des organismes de prêts peut venir buter sur des difficultés institutionnelles, souvent d'ordre politique. Sur cette base, les freins à l'essor du crédit ont surtout été conçus comme devant engendrer des différences entre entités nationales, les variations internes à un pays étant alors imputées au manque de capital social de tel ou tel marché. Dans cette perspective, lorsqu'une région a, du fait de son histoire, acquis une bonne expérience des marchés financiers, on s'attend à ce qu'elle connaisse une croissance rapide dès que de nouvelles opportunités se présentent, car les marchés financiers sont déjà implantés ${ }^{9}$. Ailleurs, à l'inverse, les mêmes opportunités ne donneront lieu à des réalisations que plus lentement, le temps que les marchés s'installent et se consolident. En outre, on souligne souvent que les marchés de crédit résultent d'une dynamique de long terme liée aux dotations locales, et donc à la richesse. L'argument est que plus une personne est riche, plus il est probable qu'elle effectue un grand nombre de transactions financières, et, comme les marchés reflètent les caractéristiques des individus qui y participent, la demande pour des organisations de crédit et pour des institutions facilitant les transactions est d'autant plus forte qu'on se trouve dans une région riche. Au contraire, les régions pauvres sont victimes d'effets de seuil. Non seulement les marchés de crédit y sont peu développés, mais l'existence d'une conjoncture favorable n'est pas suffisante pour y créer de nouveaux intermédiaires. Sans doute existe-t-il d'autres institutions qui entretiennent la confiance que le capital social, et d'autres dotations que la richesse. Mais, pour simplifier, nous nous en tiendrons ici à ces deux facteurs.

Selon la façon dont on conçoit leur interaction, on peut alors imaginer différents scénarios: quatre en fait, chacun d'eux étant lié à un ensemble particulier d'hypothèses sur les liens entre le passé et l'évolution du crédit. Le premier scénario (S1) ne donne aucun rôle à la richesse et considère que le capital social se met en place sans délai. Dans le second (S2), le capital social s'accumule aussi sans obstacle; cependant il est d'autant plus abondant que le marché est riche (à la différence de S1, le passé a donc ici sa place et les marchés dépendent de l'histoire car ils dépendent de la richesse accumulée). Au contraire, pour le troisième scénario (S3), seul importe le capital social; partant, l'histoire intervient mais pour une autre raison que dans $\mathrm{S} 2$ : plus un marché est ancien plus son capital social est important, car celui-ci ne se constitue que lentement. Enfin, selon le quatrième scénario (S4), le capital social comme la richesse ont également leur rôle. La richesse suscite, en effet, une demande de capital social; celui-ci s'établit donc plus facilement sur un marché riche, même si ce processus prend du temps et peut être contrecarré par des obstacles qui le ralentissent ${ }^{10}$.

9 - LANCE E. DAvis et RoBerT J. CuLl, International capital markets and American economic growth, 1820-1914, Cambridge, Cambridge University Press, 1994 ; ID., " Un, deux, trois, quatre marchés? L'intégration du marché du capital, États-Unis et Grande-Bretagne (1865-1913)", Annales ESC, 47-3, 1992, pp. 633-674.

10 - En fait, S4 étant une combinaison de S2 et S3, on peut imaginer différentes versions de ce scénario. Celle choisie ici suggère que richesse et capital social sont complémen- 
Chacun de ces quatre scénarios doit alors être suivi dans la durée pour qu'on puisse arbitrer entre les hypothèses différentes qu'ils supposent. Mieux, ils doivent être suivis dans la longue durée car tous envisagent une association étroite entre crédit d'hier et crédit d'aujourd'hui. Si aucun bouleversement n'intervient, les deux premiers ( $\mathrm{S} 1$ et $\mathrm{S} 2$ ) prédisent une convergence entre les marchés puisque les plus petits grandissent plus vite que les plus gros ${ }^{11}$. Concrètement, la hiérarchie des marchés tendrait alors à s'aligner sur celle de la taille des villes. Les deux derniers, en revanche, prévoient une divergence car soit l'effet direct du capital social, soit l'effet combiné de la richesse et du capital social donnent l'avantage aux marchés déjà actifs. C'est dire que le temps long est nécessaire à l'analyse. Mais il n'est pas suffisant. La raison en est que les scénarios évoqués ne réagissent pas de la même manière quand les marchés sont confrontés à un choc.

Il faut d'ailleurs préciser, car il est probable que divers types de choc (sur le crédit, sur la richesse ou encore sur les techniques) n'aient pas tous les mêmes conséquences $^{12}$. Nous nous limiterons aux décennies qui précèdent et qui suivent la Révolution française puisque celle-ci a pour résultat, entre autres conséquences, d'engendrer la crise la plus grave qu'aient connue les marchés du crédit ${ }^{13}$. Mais il s'agit d'un choc très spécifique. Sans doute l'inflation de 1792 à 1795 a-t-elle anéanti le crédit privé et fait s'évaporer une quantité d'actifs financiers. Mais, ce point mis à part, la crise politique n'a guère touché la richesse. Certains lieux ont certes souffert, des individus se sont enrichis et d'autres se sont appauvris, mais la distribution de la richesse dans le pays n'a pas été bouleversée. Reprendre nos scénarios dans ce contexte nous amène donc à préciser leurs prédictions sur le déroulement de la crise et sur ses conséquences - prédictions qui, confrontées à ce que nous savons de l'évolution des marchés, doivent nous permettre de choisir le scénario qui rend le mieux compte des effets de la Révolution.

Pendant la crise, les quatre scénarios sont unanimes: les marchés vont tous y perdre. Toutefois, S1 ne se prononce pas sur les marchés qui pâtissent le plus, car les pertes sont conçues comme purement aléatoires. Pour les trois autres, en

taires, que l'accumulation de la seule richesse ne permet pas toujours de surmonter un déficit de capital social mais que, pour un niveau de richesse donné, un capital social adéquat peut se mettre en place lentement.

11 - Parce que, pour le premier, il n'y a pas d'obstacle à la convergence, tandis que, pour S2, le délai se limite à une phase d'accumulation de richesse.

12 - Les prédictions diffèrent selon que les chocs sont positifs (pour les périodes de boom) ou négatifs (pour les périodes de crise), ou selon que les chocs négatifs sont faibles ou forts.

13 - Pour évaluer comment et dans quelle mesure se crée un marché national au $\mathrm{XIX}^{\mathrm{e}}$ siècle, nous constituons pour une centaine de marchés une suite de coupes portant sur les années 1740, 1780, 1807, 1840, 1865 et 1899. En effet, pour autant que la création d'un marché national du crédit résulte du développement de grands établissements financiers dans le dernier quart du siècle, 1840 constitue un point de départ (car les marchés sont alors très différenciés), 1865, une étape avant les nouvelles institutions, et 1899 un point d'arrivée. Mais si l'on tient compte du mouvement de centralisation à l'œuvre depuis le XVIII' siècle, il faut suivre le processus depuis l'Ancien Régime et comprendre pourquoi la centralisation amorcée a été interrompue par la Révolution. 
revanche, la crise doit toujours être plus aiguë dans les marchés au départ importants. Comme elle met à mal les institutions dont les grands marchés ont plus que d'autres besoins, ce sont ces derniers qui doivent en être les principales victimes.

Après la crise, $\mathrm{S} 1$ continue à n'accorder aucune importance au passé. Pour $\mathrm{S} 2$ et $\mathrm{S} 3$, ce sont les marchés les plus gros qui sont dans la meilleure position ${ }^{14}$. S4 les voit au contraire languir parce que les institutions nécessaires au crédit y sont très affaiblies. Enfin, quand on imagine comment se répartit l'effort de rattrapage en comparant la croissance après la crise (entre 1807 et 1840) avec l'ampleur de l'effondrement pendant la crise (entre 1780 et 1807), comme le point d'observation retenu est l'année 1807 , soit dix ans après la stabilisation monétaire, S1 considère qu'à cette date le rattrapage a déjà dû s'opérer. Puisque, pour S2, deux marchés de même richesse doivent retrouver un niveau de crédit similaire à moyen terme, on s'attend à ce que ce soit le plus éprouvé qui augmente le plus après la crise. Si l'on privilégie le capital social (S3), ce sont au contraire les marchés les moins éprouvés qui se remettent le plus rapidement car ils ont perdu moins d'institutions; le choc a donc un effet persistant et la hiérarchie des marchés ne doit pas être la même après qu'avant. Dans l'hypothèse S4, enfin, la crise affecte surtout les marchés les plus gros (et donc les plus riches). Une partie de leurs institutions ayant été détruite, ceux-ci ont plus de mal à amorcer leur reprise que les plus petits marchés. Mais à moyen terme - et le tiers de siècle qui s'écoule entre 1807 et 1840 constitue bien un laps de temps de cet ordre -, une fois qu'ils ont pu rebâtir un ensemble d'institutions, ils rattrapent leur retard. Dans ce cas, la hiérarchie qui existait à la fin de l'Ancien Régime tend donc à se reconstituer. Le tableau 1 résume ces prédictions.

Tableau 1 - Quatre scénarios et leurs prédictions sur les effets du choc financier

\begin{tabular}{|c|c|c|c|c|c|c|}
\hline & \multirow{2}{*}{$\begin{array}{c}\text { Variable } \\
\text { explicative }\end{array}$} & \multirow{2}{*}{$\begin{array}{c}\text { Variable } \\
\text { dépendante }\end{array}$} & \multirow{2}{*}{ 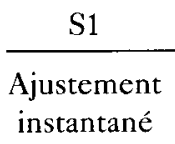 } & \multirow{2}{*}{ 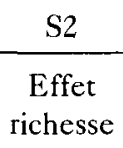 } & \multirow{2}{*}{$\frac{\mathrm{S} 3}{\begin{array}{c}\text { Effet } \\
\text { capital social }\end{array}}$} & \multirow{2}{*}{$\begin{array}{c}\mathrm{S} 4 \\
\text { Richesse et } \\
\text { capital socia }\end{array}$} \\
\hline & & & & & & \\
\hline En fin de crise & $\mathrm{C}_{\mathrm{t}-1}$ & $\Delta \mathrm{C}_{\mathrm{t}}$ & 0 & - & - & - \\
\hline À moyen terme & $\mathrm{C}_{\mathrm{t}-1}$ & $\Delta \mathrm{C}_{t+t+t}^{-t}$ & 0 & + & + & $0 /-$ \\
\hline Rattrapage & $\Delta \mathrm{C}_{\mathrm{t}-1, \mathrm{t}}$ & $\Delta \mathrm{C}_{\mathrm{t}, \mathrm{L}+1}^{\mathrm{ct+1}}$ & 0 & - & + & - \\
\hline
\end{tabular}

Notes: $\mathrm{C}_{\mathrm{t}}$ est l'activité du crédit à la date $t, \Delta \mathrm{C}_{\mathrm{t}-1, \mathrm{t}}$ la croissance du crédit entre $\mathrm{t}-1$ et $\mathrm{t}$. La date $t$ est prise pour l'année de la fin de la crise, celle de $t-1$ pour l'année avant la crise, celle de $t+1$ pour une année où les effets à moyen terme peuvent êtres perçus.

Un signe + indique que le coefficient attendu pour la variable explicative est positif; un signe - que le coefficient est négatif; un zéro indique une absence de lien.

Pour S1, la prédiction dépend de la mesure choisie. Si l'on considère le lien entre la taille des marchés avant le choc et la croissance après le choc $\left(\mathrm{C}_{\mathrm{t}-1}\right.$ par rapport à $\left.\Delta \mathrm{C}_{\mathrm{t},+1+1}\right)$, l'effet attendu est nul. Si l'on considère le lien entre la taille des marchés juste après le choc et la croissance après le choc $\left(\mathrm{C}_{\mathrm{t}}\right.$ par rapport à $\left.\Delta \mathrm{C}_{\mathrm{t} t+1}\right)$, on s'attend à un lien négatif.

14 - Pour S2, la richesse régit le développement des marchés. Les institutions s'adaptant instantanément, les marchés importants avant la crise se remettent rapidement ensuite si la richesse n'a pas été touchée. 
S'il est donc impératif que l'analyse puisse couvrir une durée assez longue, il l'est tout autant de disposer d'un découpage spatial assez fin. En effet, la richesse passée étant située dans un lieu précis, comme le sont les marchés, il nous faut observer ces derniers dans un cadre local. C'est pourquoi les marchés que nous allons étudier sont conçus comme organisés dans l'espace proche qui gravite autour d'une ville ou d'un bourg ${ }^{15}$. Pour préciser les relations entre le crédit et son environnement, nous aurons en effet à rendre compte des liens entre crédit, richesse et taille des villes. Il est banal de dire que les régions riches à un moment donné ont de bonnes institutions de crédit ou que les grandes villes sont des marchés particulièrement importants pour les prêts. Encore faut-il pouvoir cerner les liens entre richesse, urbanisation et crédit. Pour ce faire, nous mesurons le crédit par les transactions effectuées dans chaque marché et non pas à l'échelle d'une région, d'un instrument ou d'une famille, ce qui ne permettrait pas de comparer les institutions qui le soutiennent. Concrètement, nous nous appuyons sur trois coupes, une témoignant pour la fin de l'Ancien Régime (en 1780), une autre pour les lendemains immédiats de la Révolution (1807), une, enfin, plus éloignée du choc (1840). Pour chacune de ces années, nous avons relevé, marché par marché, tous les actes de crédit qui ont laissé des traces dans les registres de l'administration fiscale.

Ces exigences impliquent des contraintes. Nous avons ainsi concentré nos efforts sur les marchés du crédit notarié conçu dans un sens étroit. Comme notre information provient d'archives qui résument la plupart des contrats notariés dans un espace donné, nous pouvons couvrir une fraction très importante des transactions de crédit, non la totalité de celles-ci. En particulier, force est ici d'abandonner le crédit commercial et bancaire, qui, quand il est à court terme, nous échappe trop souvent. D'autre part, il serait a priori possible de traiter de toutes les transactions de capital : le crédit comme l'immobilier, les dots comme les apprentissages. Mais la tâche serait trop lourde et nous y avons renoncé pour ne pas renvoyer tout résultat à un lointain futur, quitte à nous interdire de traiter du problème des externalités de réseaux entre les différents marchés de capitaux. On renonce, par exemple, à analyser comment co-évoluent dans un espace donné les transferts intra-familiaux de capital (les dots en particulier) et les transferts inter-familiaux (baux, contrats de prêts) selon que les marchés du crédit sont forts ou faibles ${ }^{16}$. De même, nous nous sommes limités aux actes qui traitent de crédit exclusivement et négligeons donc les prêts qui sont partie intégrante d'autres transactions. Or ce genre de prêt est assez couramment pratiqué dans certains domaines, pour les ventes immobilières en particulier. Il se peut donc que le crédit se soit développé par d'autres canaux que ceux observés ici.

Quoi qu'il en soit, pour disposer d'informations précises sur les instruments de crédit et sur les conditions auxquelles les prêts sont accordés, il fallait collecter des renseignements prêt par prêt. C'était la condition pour étudier les liens qui unissent les marchés et les bouleversements qu'a pu y apporter la Révolution.

15 - En pratique, nous avons admis que le ressort d'un bureau de l'Enregistrement constitue une bonne approximation de cet espace. 
Face à un choc de dimension nationale comme celui-là, plusieurs possibilités sont en effet envisageables. Si le marché est unifié, le choc est unique comme le sont les réponses. Mais si les marchés sont plus différenciés (parce que l'information circule difficilement ou que les économies locales sont spécialisées), on doit s'attendre à ne pas retrouver partout les mêmes effets. Plus les marchés sont isolés, plus ils auront tendance à réagir de façon individualisée, tant ils diffèrent au départ. Il faut donc mesurer leur activité par le menu ou, à tout le moins, savoir la place relative qu'y occupent les divers types de contrats avant et après le choc. C'est ce que nous permettent nos coupes de 1780, 1807 et 1840 .

Enfin l'espace. Nous ne débordons pas le cadre national défini lors de la Révolution, admettant à ce stade que c'est là un horizon suffisant. L'historiographie ne se représente-t-elle pas les marchés du crédit de l'époque comme étant surtout cloisonnés dans des espaces moindres, soit locaux, soit régionaux? Mais, à l'intérieur de cet espace, nous voulons pouvoir étudier la géographie du crédit en détail, au moins sur certains points. Et, pour cela, il faut passer par les transactions ellesmêmes, savoir où chacune s'est traitée et où habitent les contractants. C'est à cette condition que l'on peut connaître le montant des sommes prêtées dans un marché donné, par type de marché ou encore par type de participant (agricole ou industriel par exemple). Ici, toutefois, nous ne nous attacherons qu'à quelques aspects des hiérarchies existantes. Par exemple, nous ne nous intéresserons pas à la totalité des marchés d'une province (ou d'un département), ni à la façon dont évoluent les flux de ressources entre marchés (ce qui supposerait de tirer parti des adresses des prêteurs et des emprunteurs mais aussi d'identifier les acteurs eux-mêmes). En revanche, nous avons voulu disposer d'informations sur l'activité des marchés en fonction de la taille des villes autour desquelles ils s'organisent. Or, à lui seul, ce choix est lourd de conséquences puisqu'il impose d'étudier la situation d'un grand nombre de places (voir la carte page suivante; la liste des marchés est donnée infra note 23 ${ }^{17}$. Nous souhaitons en effet savoir comment évoluent les marchés des grandes villes, des petites et même des bourgs, tout en pouvant identifier des comportements régionaux, que ceux-ci s'établissent dans le ressort d'une province, d'un département ou à quelque échelle que ce soit.

D'autres choix, de moindre portée, ont aussi dû être opérés. Les uns découlent du besoin de constituer un échantillon large. D'autres viennent de la source utilisée (les registres du Contrôle des actes puis de l'Enregistrement). On vient de le voir, nous voulons des informations sur une série de marchés répartis dans l'ensemble du pays, tout en disposant pour chacun de renseignements sur les transactions qui y sont effectuées, ne serait-ce que pour déterminer l'importance de leur activité (mesurée soit en sommant les prêts réalisés dans chacun d'eux - on parlera alors du volume de l'activité -, soit en approchant l'encours des prêts en multipliant le volume par la durée moyenne de chaque type de prêt sur cette place - on parlera 


\section{Localisation de l'échantillon}

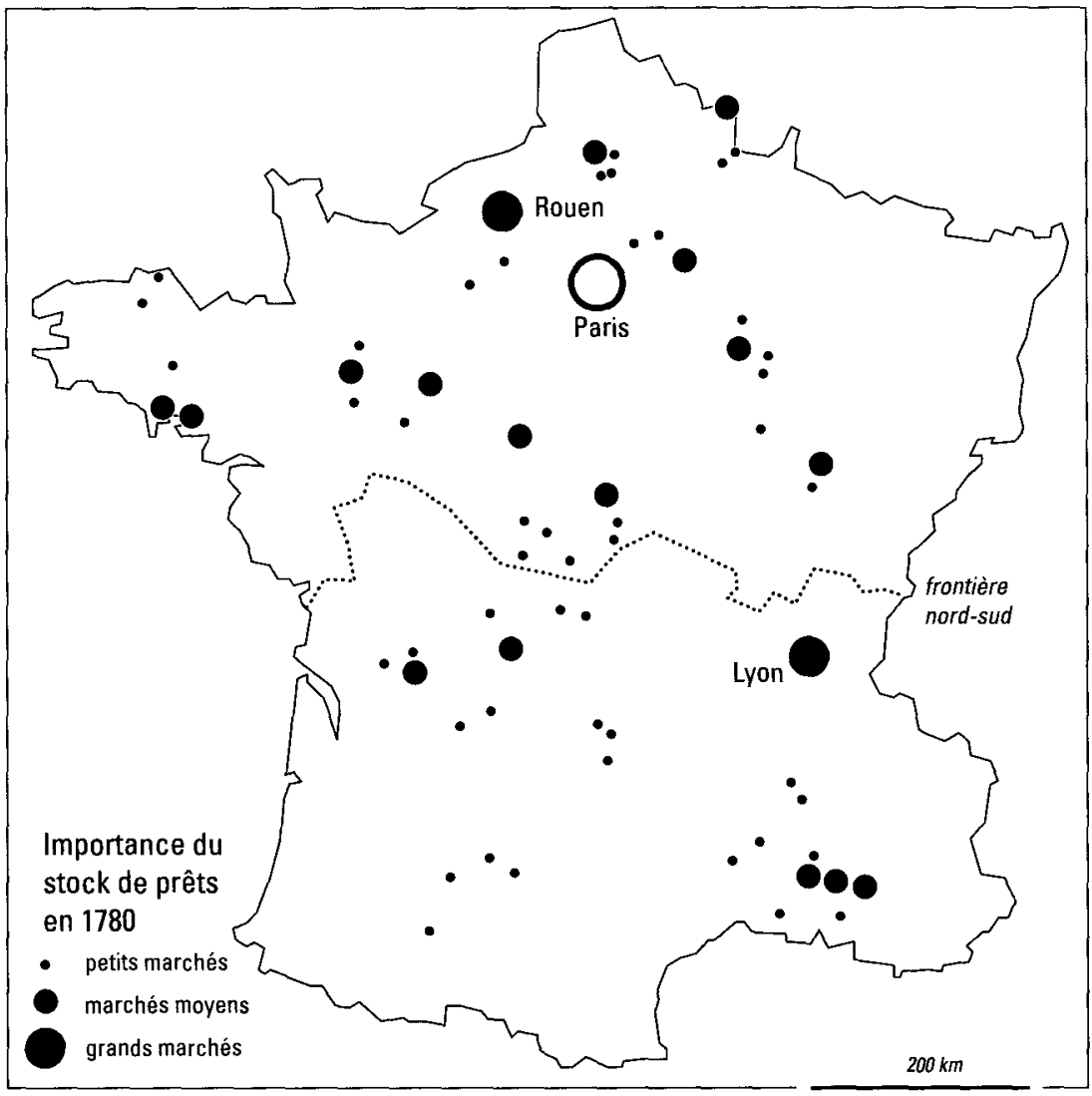

Note : Pour la liste des marchés de l'échantillon, voir note 23.

dans ce cas du stock de prêts). De la sorte, nous nous situons à mi-distance des deux historiographies sur le crédit. Nous ne pouvons nous satisfaire de résultats agrégés par marché - en reconstituant pour eux l'équivalent des agrégats que construit la comptabilité nationale sur un espace plus étendu. Une telle approche serait trop globale pour répondre aux questions qui nous préoccupent. Nous ne cherchons pas non plus à construire une base de données réunissant le maximum de renseignements sur chaque transaction, ce qui aurait imposé de recourir aux montagnes des minutes notariales passées dans chaque marché. En fait, nous sélectionnons certaines des informations sur les contrats de crédit que recensent à l'époque les registres de l'Enregistrement ${ }^{18}$. 
Nous avons renoncé aux renseignements qui ne figurent que rarement dans ces registres - ceux concernant les garanties par exemple -, mais aussi à quelques autres, pourtant toujours disponibles. En particulier, nous n'avons pas cherché ici à identifier les contractants, l'objectif étant de trouver un compromis qui permette à la fois d'aller vite (afin de pouvoir réunir des informations sur tous les actes de prêts d'un nombre assez grand de marchés) et de bien appréhender les institutions qui régissent ces marchés. Pour une transaction donnée, nous nous limitons donc aux adresses des parties et aux conditions financières des prêts.

Enfin, nous utilisons des coupes plutôt que des séries continues. La méthode a un inconvénient évident. Les années retenues font forcément abstraction des conjonctures locales et de leurs effets sur l'activité du crédit. Le biais potentiel est d'ailleurs d'autant plus grand que le marché est petit. Pourtant, on le verra, les différences qu'on observe une année donnée entre les marchés renvoient moins aux aléas qu'ils subissent à ce moment précis qu'à la variabilité des pratiques locales ou régionales. Et celles-ci restent assez stables dans le temps pour que la méthode de la coupe se révèle une approximation suffisante.

\section{La Révolution: destructions et reconstruction}

La période que couvre notre enquête est donc dominée par la Révolution française, un choc sans équivalent quant aux conséquences qu'il a eues sur les marchés considérés. Les unes ont été destructrices; d'autres, au contraire, ont été créatrices dans la mesure où la Révolution et les régimes qui lui ont succédé jusqu'à la Restauration ont mis en place un nouvel ordre du crédit qui a été conçu comme national et uniforme.

Côté destructions, d'abord. On le sait, la Révolution qui était à court d'argent dès l'automne 1789 inventa l'assignat, une monnaie papier qu'elle imprima à outrance pour survivre. L'objectif fut certes atteint, mais au prix d'une inflation vite galopante. Or, le fait que la monnaie a perdu $30 \%$ à la fin de 1792 et $99 \%$ en 1795 a provoqué un raz-de-marée dans le monde du crédit. Sous l'Ancien Régime, les prêts notariés étaient souvent à long terme et, tout aussi grave, ils étaient nominaux et à intérêt fixe. Les prêteurs subirent donc de plein fouet la dévaluation de la masse de leurs créances alors que les emprunteurs se libérèrent à peu de frais. Aussi, quand la création du franc restaura la stabilité monétaire, le crédit privé n'en continua pas moins à souffrir d'une très grave crise de confiance, d'autant que les remboursements des particuliers ont souvent coûté plus cher aux créanciers que la banqueroute des deux tiers par laquelle a été enfin réglé en 1797 le problème de la dette publique. 
Certains auteurs ont mis l'accent sur la rapidité avec laquelle l'usage de l'assignat s'est répandu dans le pays et a remis en cause les habitudes de crédit ${ }^{19}$. D'autres ont souligné que l'inflation, sans être strictement la même du nord au sud et de l'est à l'ouest, a néanmoins partout été de grande ampleur et partout dévastatrice ${ }^{20}$. D'autres encore ont insisté sur l'intensité du phénomène de désendettement ${ }^{21}$. Ajoutons ici que les effets de l'inflation se sont distribués dans l'espace de façon moins régulière qu'on pourrait le croire. Une raison en est que, d'une place à l'autre, les individus n'utilisaient pas toujours les mêmes contrats. Sous l'Ancien Régime, là où la rente constituée dominait, l'emprunteur (ou ses héritiers) était seulement tenu de verser l'intérêt, libre à lui de choisir le moment où il rembourserait le capital - après quelques années, une décennie ou un siècle, voire plusieurs. Au contraire, là où l'obligation régnait, le terme du contrat était fixé et la dette courait rarement plus d'un an ou deux. Il est aussi des régions mixtes, où rentes et obligation coexistaient. Dans ces conditions, les créanciers qui avaient placé leur argent en rente avant la Révolution étaient beaucoup plus exposés à l'inflation que ceux qui avaient accepté des obligations. Ainsi le désendettement à bon compte a-t-il frappé plus fortement là où la rente l'emportait sur l'obligation. Pour autant, la géographie des contrats ne se calque pas exactement sur la géographie de la richesse de sorte que, si les régions à rentes étaient souvent riches, les régions à obligations étaient loin de se confondre avec les régions pauvres. Sur cette base, on peut avancer deux hypothèses supplémentaires sur le devenir des marchés du crédit entre 1780 et 1840 . Il est probable, en effet, que les marchés dont l'histoire sous l'Ancien Régime a conduit à privilégier les rentes ont eu du mal à se remettre de la Révolution car la confiance y a été plus qu'ailleurs détruite. En même temps, là où cette destruction a eu lieu, il se peut qu'elle ait facilité l'acceptation de nouvelles institutions.

Car si la Révolution a détruit, elle fut aussi une période d'intense activité législative et les mesures prises alors concernèrent, pour la première fois, le pays dans son entier. En matière de crédit, c'est le cas en particulier de l'abolition de la législation sur l'usure ou de la généralisation du système d'information publique sur la situation des gages qui se mit en place avec la réforme de l'administration des Hypothèques. La révision du Code civil et du Code du commerce eut aussi une grande importance. Enfin, la consolidation de la dette publique et la création de la Banque de France furent deux innovations qui auraient pu permettre

19 - Eugene N. Whrte, «Measuring the French Revolution's inflation: the Tableaux de dépréciation ", Histoire et mesure, 6-3/4, 1991, pp. 245-274.

20 - ANDREw Dickson White, Fiat money inflation in France, New York-Londres, D. Appleton/Century company incorporated, [1896] 1933; Pierre Garon, Tableaux de dépréciation du papier-monnaie, Paris, Imprimerie nationale, 1909; Seymour Harris, The assignats, Cambridge, Harvard University Press, 1930.

21 - Michel Vovelle, Ville et campagne au XVIII ${ }^{e}$ siècle. Chartres et la Beauce, Paris, Éditions sociales, 1980; GILlES POSTEL-VINAY, La terre et l'argent. L'agriculture et le crédit en France du XVIII ${ }^{e}$ au début du XX $x^{e}$ siècle, Paris, Albin Michel, 1998; Phil.tP T. Hoffman, Gilles du crédit à Paris, 1662-1869, Paris, Éditions de l'EHESS, 2001. 
l'émergence de nouvelles formes de crédit dans l'ensemble du pays. Pourtant, quelle qu'ait été la volonté des législateurs de créer un régime légal commun et des institutions nationales, force est de reconnaître que les nouvelles institutions ne se sont développées ni en tous lieux ni au même rythme. Ainsi, pendant la période considérée ici, les Hypothèques n'atteignirent guère qu'une petite fraction de la population. Le service existait sans doute, mais il était trop cher. Pour la grande majorité des biens ruraux en particulier, il était hors de prix et n'a pas été employé. De même, la Banque de France était, à l'époque, surtout la banque de Paris.

Durant les années qui suivirent la stabilisation monétaire de 1797, les marchés du crédit furent donc marqués à la fois par la peur du retour de l'inflation et par la tentation d'aller de l'avant. Mais, d'une place à l'autre, ces deux sentiments n'eurent pas toujours le même poids. Pour saisir comment se situent de ce point de vue les marchés de notre échantillon, nous devions choisir une date. En retenant l'année 1797, par exemple, on aurait mesuré la situation des marchés du crédit lorsqu'ils touchèrent le fond, au lendemain de l'hyperinflation. Un tel choix aurait cependant été peu pertinent car, alors, tous les marchés se ressemblent: comme il n'y a guère de diversité d'une place à l'autre, on n'apprendrait rien à les comparer, si ce n'est qu'il existe partout un petit nombre d'entrepreneurs que leurs "esprits animaux" poussent au risque et qui recommencent très vite à prêter. Mais, pour l'essentiel, la situation est partout homogène: partout, les créanciers avaient peur et le crédit avait donc partout quasiment disparu. De plus, la demande de crédit a chuté, elle aussi, puisque, en annulant les dettes, la Révolution a temporairement éliminé le besoin de se refinancer. Et, de fait, à en juger par les marchés dont on connaît l'activité à cette date, les transactions sont alors réduites à presque rien. Ce n'est du reste guère surprenant; et, à vrai dire, une fois constaté cet effondrement, peu importe de savoir que les stocks de prêts ont chuté, ici, de $80 \%$, là, de 90 ou de $99 \%$.

Il est plus intéressant de mesurer les effets du choc révolutionnaire en examinant la situation des marchés après un laps de temps suffisant pour que les individus et les institutions aient eu la possibilité de s'adapter. On utilisera pour cela deux coupes successives, à une génération de distance, l'une en 1807 , soit une décennie après la fin de l'inflation, et l'autre, trente ans plus tard, en 1840. Se dégagent ainsi deux conséquences de la Révolution. D'une part, le crédit notarial qui se reconstitue au XIX ${ }^{e}$ siècle est beaucoup moins centralisé que sous l'Ancien Régime. Le lien entre urbanisation et crédit est nettement plus faible après 1789 qu'avant - une situation qui dura un demi-siècle au moins. D'autre part, il se différencie de plus en plus dans l'espace. Dans la moitié Nord du pays, le crédit se reconstruit et s'étend en dépit des performances médiocres de la capitale alors que dans les campagnes de la moitié Sud les marchés s'enferment dans des structures informelles sans avenir.

Appréhender la décentralisation devient possible, dès lors que l'on dispose d'estimations sur le crédit dans suffisamment de marchés en 1780, en 1807 et en 1840. Commençons par un constat simple en distinguant quatre ensembles: Paris, 
les très grandes villes (Marseille, Lyon, Bordeaux, Rouen, Nantes), les autres villes de plus de 10000 habitants et le reste du pays ${ }^{23}$. La valeur moyenne des prêts traduit bien la hiérarchie existante (tableau 2). En 1780, celle-ci tourne autour de 300 livres sur les petits marchés mais dépasse 15000 livres à Paris. Sans doute y-a-t-il des surprises. Il arrive ainsi que sur certains petits marchés les prêts soient d'un montant assez élevé. Mais, en général, la hiérarchie des populations urbaines se retrouve de façon exponentielle dans les prêts. Entre 1780 et 1840, par contre, le prêt moyen évolue de façon très différenciée d'un marché à l'autre. De plus, deux autres composantes de l'activité de crédit varient également: le nombre de prêts par habitant et la durée des prêts. Aussi, sauf indication contraire, pour mesurer le degré d'activité des marchés nous préférerons nous référer non à la valeur des prêts mais aux stocks (la valeur des prêts multipliés par leur durée initiale). Cependant, pour donner un premier aperçu de l'histoire des marchés de 1780 à 1840 et présenter ces différents indicateurs, considérons d'abord à la fois le nombre de prêts, les sommes annuellement prêtées et les stocks existant dans chacun des grands ensembles évoqués: Paris, les grandes villes, les autres villes de plus de 10000 habitants et le reste du pays ${ }^{24}$.

Tableau 2 - Distribution des prêts notariés en France $(1780,1807,1840)$

\begin{tabular}{lrrrrrrrrrr}
\hline & \multicolumn{3}{c}{$\begin{array}{c}\text { Nombre de prêts } \\
\text { (pour 1 000 hab.) }\end{array}$} & \multicolumn{3}{c}{$\begin{array}{c}\text { Valeur des prêts } \\
\text { (par hab.) }\end{array}$} & \multicolumn{3}{c}{$\begin{array}{c}\text { Stock des prêts } \\
\text { (par hab.) }\end{array}$} \\
& 1780 & 1807 & 1840 & 1780 & 1807 & 1840 & 1780 & 1807 & 1840 \\
\hline Paris & 15,0 & 12,4 & 7,3 & 238,7 & 177,7 & 123,0 & 1918,3 & 781,6 & 695,8 \\
Grandes villes & 10,7 & 15,6 & 8,2 & 39,9 & 69,8 & 63,6 & 342,3 & 360,5 & 316,8 \\
Autres villes & 10,7 & 15,1 & 22,2 & 8,9 & 17,0 & 40,4 & 81,7 & 53,8 & 162,4 \\
Reste & 13,1 & 15,4 & 17,3 & 5,0 & 8,1 & 14,1 & 33,9 & 23,2 & 48,4 \\
\hline & \multicolumn{1}{c}{ Total } & \multicolumn{4}{c}{ Total (en milliers) } & Total (en milliers) \\
\hline Paris & 9046 & 7196 & 6591 & 144201 & 103074 & 111808 & 1158668 & 453322 & 632445 \\
Grandes villes & 4856 & 7757 & 4515 & 18107 & 34628 & 35084 & 155401 & 178796 & 174858 \\
Autres villes & 19785 & 27507 & 44632 & 16408 & 30943 & 81055 & 151352 & 98098 & 325952 \\
Reste & 327862 & 444285 & 591200 & 125522 & 233468 & 480804 & 851525 & 666535 & 1652259 \\
\hline France & 361549 & 486745 & 646938 & 304238 & 402113 & 708751 & 2316946 & 1396751 & 2785514 \\
\hline
\end{tabular}

Note: Les valeurs et les stocks des prêts sont en livres, puis en francs.

23 - Les grandes villes sont représentées par Lyon et Rouen; les autres villes de plus de 10000 habitants par Amiens, Montpellier, Avignon, Limoges, Troyes, Bourges, Dijon, Montauban, Le Mans, Laval, Angoulême, Châteauroux, Blois, Vannes, Périgueux, Aurillac, Moissac et Évreux; et le reste du pays par Mayenne, Orange, Saint-Amand, La Flèche, Pontivy, Lectoure, Maubeuge, L'Isle-sur-la-Sorgue, Château-Gonthier, Apt, Salon, Saint-Gilles, Guéret, Château-Thierry, Buzançais, La Châtre, Saint-Jean-du-Gard, Privas, Dun-sur-Auron, Auray, Bellac, Montignac, Mauriac, Tréguier, Saint-Ambroix, Nuits-Saint-Georges, Hirson, Arcis-sur-Aube, Villers-Cotterêts, Vervins, Mirande, Corbie, Rosières, Bar-sur-Seine, Jarnac, Montbard, Rugles, Moreuil, Excideuil, Vendeuvre, Salers, Belle-Isle-en-Terre, Chénerailles, Rochemaure et Ailly-sur-Noye. Les villes sont ici classées par ordre décroissant de population au recensement de 1836; le montant moyen des stocks est calculé en pondérant le stock moyen par tête dans chaque sousensemble par le poids de la population de ces sous-ensembles dans la population totale. 24 - Les résultats obtenus ici à partir d'un échantillon élargi modifient quelque peu nos évaluations précédentes sans en changer les conclusions sur l'ampleur du choc et la 
Le constat est net: si, de façon générale, les marchés du crédit ont beaucoup souffert de la Révolution, celui de la capitale apparait comme la principale victime. Certes, d'autres déclinent plus encore - comme celui de L'Isle-sur-la-Sorgue par exemple puisque, avec la Révolution, ce marché perd le statut de petite Suisse locale que lui avait valu sous l'Ancien Régime sa qualité d'enclave papale. Mais, hormis ce genre de cas, des quatre groupes de marchés distingués, c'est celui de la capitale qui est le plus atteint. Alors qu'il concentrait près de $50 \%$ du stock de crédit notarié en 1780 , sa part tombe à $33 \%$ en 1807 et $23 \%$ en 1840 . Globalement, les grandes villes font à peine mieux ${ }^{25}$. En fait, ce sont les marchés des petites villes et ceux du monde rural qui ont assuré le rétablissement du crédit (graphique 1).

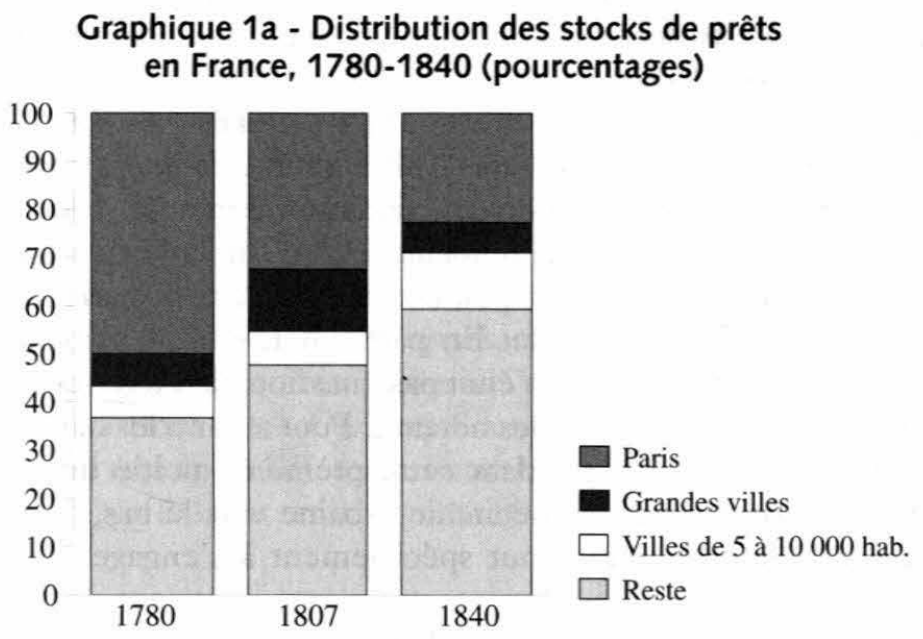

\section{Graphique 1b - Distribution des stocks de prêts en France, 1780-1840 (montants*)}

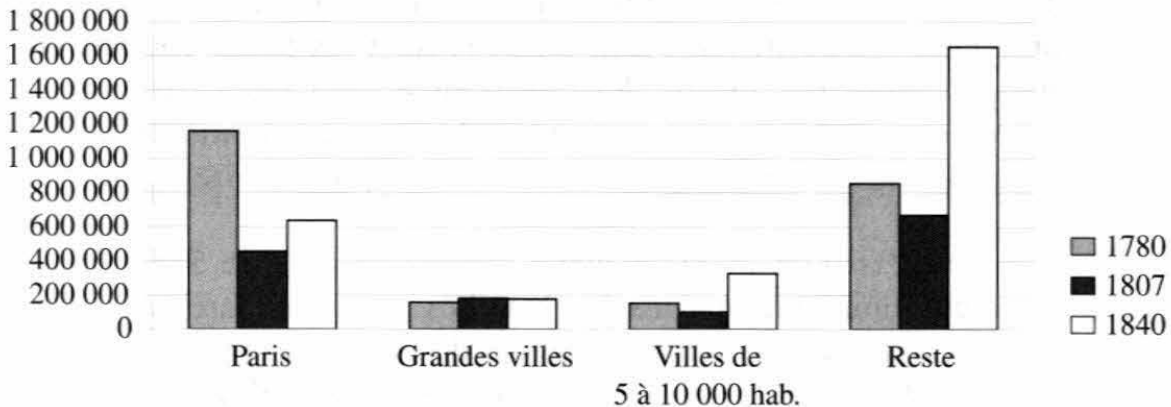

* Les montants sont en milliers de livres, puis de francs.

redistribution spatiale du crédit (Philip T. Hoffman, Gilles Postel-Vinay et JeanLaurent Rosenthal, «Économie et politique. Les marchés du crédit à Paris, 17501840 », Annales HSS, 49-1, 1994, pp. 65-98, et ID., Des marchés sans prix..., op. cit., p. 310. 25 - On notera cependant que Lyon et Rouen suivent des évolutions assez différentes. 
Précisons, en examinant successivement nos différents indicateurs, et d'abord le nombre des prêts. Ce critère a le double intérêt de faire ressortir la place prédominante du monde rural pendant la période et de rappeler que, pour maintenir l'encours à un niveau constant, le nombre de prêts doit beaucoup augmenter quand la durée moyenne des prêts chute. Or c'est ce qu'on observe dans les décennies qui suivent la Révolution (du fait notamment de la disparition des rentes). Du moins, c'est bien ce qui se produit dans le monde rural et les petites villes. Mais tel n'est pas le cas dans les grandes villes et cette différence persiste jusqu'en 1840. Le volume des prêts, lui aussi, a tendance à croître à la campagne de 1780 à 1807 , puis partout après cette date. Enfin, si l'on considère les stocks, la décentralisation est brutale: en 1840, les marchés de moins de 10000 habitants concentrent en effet près de $60 \%$ du crédit notarié. Mais leur croissance pendant les décennies post-révolutionnaires est en fait un peu moins rapide que celle des marchés des villes de 10000 à 75000 habitants.

La Révolution eut donc deux effets sur les marchés du crédit. Elle réduisit les stocks de crédit notarié de $40 \%$ de 1780 à 1807 et elle les redistribua dans l'espace. Après un fort mouvement de concentration durant la seconde moitié du $\mathrm{XVIII}^{\mathrm{e}}$ siècle, la Révolution inversa la tendance et créa un crédit de proximité. Cet effet tendit ensuite à s'effacer peu à peu et avait souvent disparu en 1840. Mais, là encore, la règle n'est pas générale. En particulier, elle ne s'applique pas aux grandes villes. Pour ces dernières, il n'était pas question de retourner à ces intermédiaires d'Ancien Régime qu'étaient les notaires. Pour autant, les banques n'étaient pas capables d'assurer le relais pendant cette première moitié du $\mathrm{XIX}^{\mathrm{e}}$ siècle. $\mathrm{Si}$ elles se diffusèrent du haut de la hiérarchie urbaine vers le bas, leur progression resta sélective et elles hésitèrent tout spécialement à s'engager dans des prêts hypothécaires.

Cette décentralisation du crédit est d'autant plus surprenante que l'une des réformes de la Révolution en matière de crédit a consisté à $\operatorname{créer}^{26}$ un système d'information sur les gages immobiliers qui, en pratique, a surtout bénéficié aux riches ou du moins aux détenteurs d'immeubles importants. En effet, les Hypothèques ont adopté une tarification qui, en pénalisant les petits contrats courts, était doublement régressive puisque les gros prêts étaient longs par rapport aux petits. Ce système qui se mit en place pendant la première moitié du XIX ${ }^{\mathrm{e}}$ siècle n'était donc à la portée que de gros emprunteurs, propriétaires d'immeubles importants.

Comme les prêts d'un montant élevé (disons de plus de 5000 francs) se négociaient exceptionnellement dans les marchés ruraux et rarement dans les petites villes, la réforme de l'hypothèque aurait dû conforter la concentration des prêts dans les grandes villes et surtout dans la capitale. Or tel n'a pas été le cas, bien au contraire. Force est donc de conclure qu'entre l'espoir lié aux réformes et la peur qui continuait à tenailler les créanciers depuis la Révolution, c'est la peur 
qui l'emporta. Si l'on prêtait, c'était à proximité, comme si les créanciers trouvaient plus rassurant que leurs partenaires habitassent le même arrondissement, le même canton, voire la même commune. Dans ces conditions, ce sont les petits prêts ruraux et ceux négociés dans les petites villes (d'ailleurs souvent destinés à des ruraux) qui assurèrent le redémarrage du crédit notarié. Et comme les (petits) prêts ruraux n'allaient pas aux citadins qui avaient été par le passé les grands bénéficiaires du crédit, la décentralisation se doubla d'une réorientation des ressources. La Révolution semble donc avoir durablement augmenté les coûts de transaction du crédit à distance et avoir profondément modifié la relation du crédit à la hiérarchie urbaine.

À cette décentralisation s'ajoute une autre transformation, dont la portée n'est pas moindre: le crédit se régionalisa selon un découpage spatial particulier. Alors que la Révolution tenta d'inventer une nation de citoyens égaux face aux institutions, les marchés du crédit s'autorisèrent des manies qui tantôt ne s'imposaient que sur une place ou dans une région et tantôt s'étendaient plus largement. Tenons-nous en ici à ces différenciations qui couvraient des espaces assez vastes.

Dès 1807 et plus encore en 1840, deux France se distinguent nettement en matière de crédit. Au Nord, le crédit est long et circule presque exclusivement sous forme d'obligations. Au Sud, il est court et une gamme de contrats est peu à peu mise en place. Le graphique 2 présente la distribution des types de contrats par marché en 1780, 1807 et 1840, en opposant une France du Nord et une France du Sud dont la frontière semble suivre un tracé un peu tremblé le long d'une ligne allant de La Rochelle à Genève. Sous l'Ancien Régime, le graphique 2 le montre, il existait une vraie différence entre le Sud (où les obligations étaient plus fréquentes) et le Nord (où la rente dominait). La part du volume des prêts qui fait

\section{Graphique 2 - Distribution des prêts avant et après la Révolution}

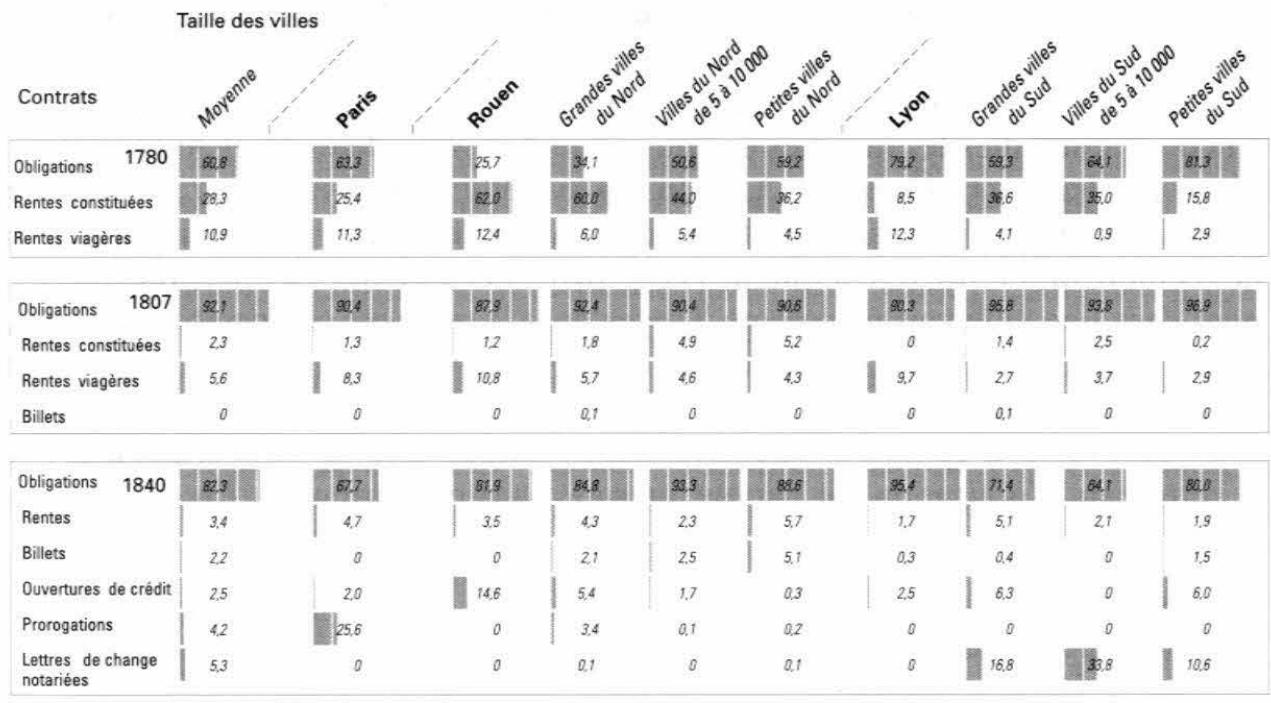


l'objet de contrats de rente est ainsi presque deux fois plus forte dans le Nord que dans le Sud (une régression confirme que cette différence est significative).

\section{Tableau 3 - Poids de certaines variables susceptibles d'expliquer le déclin des stocks de prêts entre 1780 et 1807}

\begin{tabular}{lcccc}
\multicolumn{1}{c}{ Variables explicatives } & Coefficient & $\mathrm{t}$ de Student & Coefficient & $\mathrm{t}$ de Student \\
\hline Constante & 0,046 & 3,17 & 0,013 & 1,25 \\
Part des rentes en 1780 & $-0,024$ & $-1,4$ & $-0,044$ & $-2,65$ \\
Log du stock des prêts en 1780 & $-0,007$ & $-2,9$ & & $-0,32$ \\
Log de la population urbaine & & & $-0,001$ & 0,47 \\
Nord/Sud & 0,003 & 0,34 & 0,004 & \\
\hline $\mathrm{R}^{2}$ & 0,21 & & 0,11 & \\
$\mathrm{R}^{2}$ ajusté & 0,18 & & 0,06 & \\
Écart type & 0,031 & & 0,033 & \\
Nb d'observations & 65 & & 65 & \\
\hline
\end{tabular}

Note : La variable «Nord/Sud » $=1$ si le marché est dans le Nord, 0 s'il est dans le Sud.

Si nette soit-elle, cette hétérogénéité ne se réduit pas à une opposition macrorégionale entre Nord et Sud. En fait, elle se retrouve aussi à l'intérieur même des régions (au sens courant du terme) ${ }^{27}$. Ainsi, dans la Bretagne septentrionale, les marchés de Tréguier et de Belle-Isle-en-Terre se ressemblent et font une large place à l'obligation, tandis que, sur la côte Sud, à Vannes et à Auray, la rente l'emporte. De même, dans le Sud-Ouest, les marchés situés dans l'actuel département du Gers marquent un appétit très particulier pour les rentes, alors que leurs voisins à Montauban et Moissac font une grande consommation d'obligations. On pourrait multiplier les exemples mais l'essentiel est de souligner combien les idiosyncrasies locales étaient fortes en 1780 . Le fait n'est d'ailleurs pas surprenant. Même si la crise qui frappa le crédit à la fin du règne de Louis XIV et pendant la Régence a pu avoir un effet unificateur, bien des décennies s'écoulèrent ensuite au cours desquelles les marchés ont pu suivre des voies diverses et se spécialiser d'une manière ou d'une autre. À Dijon, où le crédit était dominé par les états de Bourgogne et les officiers des institutions provinciales (états, parlement, chambre des comptes), la rente tenait une très large part. Au contraire, les obligations dominent dans une ville voisine comme Troyes dont la taille est comparable mais qui, moins peuplée d'officiers et privée d'assemblée provinciale, était surtout un centre commercial et industriel.

1807 rompt avec cette différenciation régionale, comme l'indique le graphique 2. À cette date, l'obligation est partout presque sans concurrents ${ }^{28}$. En ce sens, on

27 - Une régression (non donnée dans les tableaux) de la part des rentes en 1780 sur une variable indicatrice (Nord $=1$, Sud $=0$ ) et la population du marché n'explique qu'un tiers de la variance.

28 - Une régression de la part des obligations en 1807 sur une variable indicatrice (Nord $=1$, Sud $=0$ ) et la population du marché ne donne aucun coefficient significatif. 
peut dire que la Révolution a gagné son pari : le crédit est uniforme, au moins pour ce qui est des contrats. Seule leur durée varie. C'est même là que s'est réfugiée la différence Nord-Sud. À population égale, un marché dans le Nord avait des prêts qui duraient en moyenne vingt mois de plus que dans le Midi, à un moment où la durée moyenne des prêts méridionaux n'était que de deux ans et demi. Mais, résiduelle en 1807, la régionalisation du crédit s'accentue ensuite. En 1840, la part des obligations dans le volume des prêts varie de 64 à $80 \%$ sur les marchés organisés autour des villes petites et moyennes du Sud, alors qu'elle tourne autour de $90 \%$ dans le Nord. ${ }^{29}$. Encore faut-il préciser. Si le régime de l'obligation à tout faire domine au Nord, il laisse une certaine place à d'autres instruments. Par exemple, l'usage du billet à ordre est fréquent dans le Bassin parisien (d'Amiens à Dijon et de Blois à Bourges). De courte durée et utilisé pour de petites sommes, ce contrat resta toutefois marginal et pourrait être négligé s'il n'avait pas, dans le Sud, un pendant dont le succès est vite beaucoup plus marquant.

Dans le Midi, en effet, les obligations ont peu à peu cédé du terrain à un autre type d'acte: la lettre de change notariée. Qui dit lettre de change, dit crédit marchand et donc des prêts pour lesquels nul n'a besoin de notaire. Pourtant ce cheval à cinq pattes joue un grand rôle sur de nombreux marchés : parfois, comme à Saint-Gilles-du-Gard ou à Moissac, il supplante même l'obligation. À sa façon, cet instrument nouveau constitua une réponse aux craintes qui hantaient les prêteurs depuis la Révolution. C'est un contrat court, local et dont le taux pouvait dépasser le plafond de $5 \%$ qu'une loi de septembre 1807 avait rétabli pour les obligations. Absent dans la coupe 1807, ce contrat se diffuse rapidement dans la moitié Sud du pays. Dans le Nord, au contraire, sur plus de 18000 contrats relevés en 1840, on ne trouve de lettres de change notariées qu'à l'état de traces (une à Dijon, neuf à Rugles, trois à La Châtre...).

La description de ces trois coupes permet donc de dégager plusieurs résultats qu'il importe maintenant d'expliquer. La Révolution joua d'abord un rôle destructeur. Il est sûr que sans elle le crédit notarié se serait mieux porté en France en 1807 mais aussi en 1840. À cette date encore, si la reconstitution des circuits de crédit peut être considérée comme acquise dans les campagnes, elle reste inachevée dans les grandes villes. Ensuite, la Révolution créa ; ainsi parvint-elle, en partie du moins, à effacer les différences entre le Nord et le Midi. Enfin, les effets d'agglomération, d'habitude si nets dans le domaine du crédit, tendirent à disparaître pendant et immédiatement après la crise. La hiérarchie des marchés fut remise en question par l'instabilité politique avec pour effet la disparition de près de la moitié des plus gros actes de prêt (ceux de Paris et des grandes villes) et la redistribution du crédit notarié vers des petits prêts dans de petites villes et à la campagne. 


\section{Les institutions et l'évolution du crédit, 1780-1840}

Pour expliquer ces changements, revenons aux scénarios évoqués plus haut pour distinguer comment la Révolution affecta les marchés et comment ceux-ci tentèrent de s'en remettre. On l'a vu, divers mécanismes peuvent contribuer à assurer le succès des transactions. Le plus évident est la confiance : les choses ne se passent pas de la même manière selon qu'aux yeux des créanciers prêter paraît sûr, risqué ou tout à fait fou. Jouent également les institutions formelles (législation, contrats privés dont la mise en œuvre peut être imposée par voie de justice...) comme les institutions informelles (contrats implicites entre prêteurs, emprunteurs et intermédiaires...). Selon que l'un ou l'autre de ces mécanismes est présent, on peut imaginer que le développement des marchés s'en trouve affecté (par exemple, il est classique de se demander dans quelle mesure l'absence de mécanisme permettant aux banques de réduire leurs réserves a limité leur essor). On peut aussi imaginer que, après la Révolution, ces mécanismes se reconstituèrent très vite (S1) ou qu'il leur fallut du temps (S2), voire beaucoup de temps (S3 et S4). Ce qui est en cause, c'est le poids du passé dans la croissance du crédit.

Or nos scénarios permettent une formulation simple des liaisons entre crédit passé et croissance des prêts. $\grave{A}$ une condition toutefois: tels ou tels d'entre eux peuvent avoir des prédictions identiques sur certains intervalles de temps. Par exemple, S2 et S3 ont la même prédiction sur l'effet à moyen terme d'une crise; en revanche, ils divergent sur les capacités de rattrapage dans le moyen terme. Il faudra donc les mettre à l'épreuve sur plusieurs coupes pour pouvoir décider lequel retenir. Admettons alors que les stocks de crédit soient le fruit des histoires locales et que la croissance du stock de prêts entre deux dates dans un lieu $\mathrm{i}, \Delta \mathrm{C}_{\mathrm{i}}$, soit une fonction directe du stock initial de prêts dans ce lieu : $\Delta \mathrm{C}_{\mathrm{i}}=\alpha+\beta \mathrm{C}_{\mathrm{i}}+\gamma \mathrm{X}_{\mathrm{i}}+\varepsilon$, où le vecteur $\mathrm{X}_{\mathrm{i}}$ est une série de variables sur les environnements locaux ${ }^{30}$.

Pour le choc de la Révolution, l'intervalle considéré est délimité par les années 1780 et 1807 (dans le tableau 1, de t-1 à t). Pour cette période, comme on l'a vu plus haut, S1 prédit un coefficient $\beta$ nul, S2, S3, et S4 un coefficient négatif. La phase de reconstruction à moyen terme après la crise est, elle, délimitée par les années 1807 et 1840 (dans le tableau 1, de $t$ à $t+1$ ). Pour cette période, $\mathrm{S} 1$ prévoit un coefficient nul, S2 et S3 un coefficient positif, S4 un coefficient négatif.

En cherchant à capturer ces effets, il faut certes veiller à ne pas introduire de biais statistique. Le risque peut, en particulier, provenir des aléas de mesure du crédit. Ainsi, le stock de prêt de 1780 intervient ici à la fois comme variable explicative et dans le taux de croissance de 1780 à 1807 . Si les stocks de prêts en 1780 sont gonflés pour une raison aléatoire, il en résultera donc un biais négatif sur le coefficient. Une solution peut être alors de remplacer le stock des prêts en

30 - Cette équation ne repose pas sur un modèle économique précis. Elle peut être reliée à différentes versions de la croissance endogène mais notre but n'est ici qu'une description systématique de nos données. 
1780 par la population des villes à la même date. Cette approche se justifie ici en raison des liens étroits entre la taille des villes et l'activité de crédit ${ }^{31}$. De plus, les aléas de mesure des populations urbaines n'ont pas de liens avec les aléas de mesure des prêts. Utiliser la population comme instrument est toutefois problématique car c'est supposer que toute variation du stock de prêts entre marchés de même taille est l'effet non de leur spécialisation respective mais de purs aléas de mesure alors que, précisément, pour S2, S3 et S4, ces variations renvoient aux caractéristiques structurelles des marchés et sont liées à la richesse et/ou au capital social. Pour S2, par exemple, un marché riche aura plus de crédit qu'un marché pauvre à population égale. Et il en ira de même, dans le scénario S3, pour les marchés à fort capital social. De plus, pour la coupe de 1780, qui saisit la situation des marchés après une longue période de (relative) stabilité financière, il est probable que les effets de richesse et de capital social aient été spécialement forts et que, de ce fait, l'instrument soit inadéquat. Au contraire, en 1807 , après le choc de la Révolution, on s'attend à ce que la hiérarchie des villes et celle du crédit soient très proches. En d'autres termes, la population est probablement un meilleur instrument pour 1807 que pour 1780. C'est pourquoi nous présentons des régressions qui tiennent compte soit du stock de prêts, soit de la population.

Celles-ci montrent que plus un marché était actif sous l'Ancien Régime, plus il fut atteint par la Révolution. Le coefficient du stock de prêts en 1780 est négatif et statistiquement significatif dans la régression sur la variation des stocks de prêts 1780-1807 (tableau 3) ${ }^{32}$. Le coefficient de la population en 1780, lui, n'est pas significatif. Compte tenu du fait que la corrélation entre la population des villes et le stock de prêts est très forte en 1780 et $1807^{33}$, c'est dire que les marchés qui ont le plus souffert étaient ceux qui étaient les plus actifs à population donnée. Ce constat peut avoir deux explications. L'une, banale, évoquerait un phénomène de retour à la moyenne: les aléas du crédit observés en 1780 se seraient effacés avec le temps. Mais il est aussi possible que les différences entre marchés à la fin de l'Ancien Régime, loin d'être des aléas momentanés, aient reflété des écarts durables liés à des institutions différentes. Dans cette mesure, parce qu'elle a détruit ces institutions, la Révolution a surtout nui aux marchés les plus forts. Si l'on retient cette seconde hypothèse, on est conduit à écarter le scénario S1 (ce que va confirmer l'analyse de la période suivante).

Il reste alors à trancher entre les autres scénarios. Pour cela, il faut un observatoire temporel plus long. Considérons donc le laps de temps de trois décennies qui suit la crise et constitue une période de croissance des marchés du crédit (1807-1840). Là, le stock de prêts en 1807 comme la population du

31 - Dans des régressions non reportées ici, on observe bien une relation forte entre l'importance de la population urbaine et l'activité de crédit pour chaque année considérée. 32 - Dans les régressions qui suivent, la variation des stocks entre deux dates est définie comme un taux de croissance annuel.

33 - Elle est de 0,84 à ces deux dates si l'on exclut le marché parisien et serait plus forte encore si l'on prenait en compte le cas extrême de la capitale. 
marché à cette date jouent négativement sur la variation des stocks de prêts entre 1807 et 1840 (tableau 4). C'est là un résultat suffisant pour écarter les scénarios S2 et S3.

\section{Tableau 4 - Poids de certaines variables susceptibles d'expliquer la croissance des stocks de prêts entre 1807 et 1840}

\begin{tabular}{lcccc}
\hline \multicolumn{1}{c}{ Variables explicatives } & Coefficient & $\mathrm{t}$ de Student & Coefficient & $\mathrm{t}$ de Student \\
\hline Constante & 0,038 & 4,65 & 0,021 & 4,25 \\
Nord/Sud & 0,024 & 5,09 & 0,025 & 5,03 \\
Part des rentes en 1780 & $-0,004$ & $-0,04$ & $-0,011$ & $-1,13$ \\
Log du stock des prêts en 1807 & $-0,004$ & $-3,03$ & & \\
Log de la population urbaine & & & $-0,003$ & $-1,77$ \\
Croissance des stocks 1780-1807 & $-0,47$ & $-7,21$ & $-0,51$ & $-7,17$ \\
\hline $\mathrm{R}^{2}$ & 0,63 & & 0,63 & \\
$\mathrm{R}^{2}$ ajusté & 0,61 & & 0,6 & \\
Écart type & 0,015 & & 0,015 & \\
Nb d'observations & 64 & & 64 & \\
\hline
\end{tabular}

Note: Voir tableau 3.

Une variable explicative supplémentaire - le taux de croissance du crédit de 1780 à 1807 - permet de savoir si l'essor des prêts de 1807 à 1840 découle des efforts faits pour surmonter la crise de la Révolution ou s'il est, tout simplement, indépendant du passé (tableau 4). De la sorte, on peut mieux arbitrer entre nos scénarios (puisque $\mathrm{S} 3$ suggère que les marchés qui ont le moins souffert sont ceux qui ensuite croissent le plus vite, alors que S1 veut ignorer cet effet du passé et que $\mathrm{S} 2$ et $\mathrm{S} 4$ prévoient un rattrapage des marchés les plus touchés). Or le coefficient de cette variable est élevé (et très significatif), de l'ordre de 0,5 . En d'autres termes, chaque fois qu'un marché voit son déclin annuel entre 1780 et 1807 excéder de $1 \%$ le déclin moyen annuel de tous les marchés, il tend à récupérer un demi-point de croissance entre 1807 et 1840 . Il faut donc préférer S4 aux autres scénarios.

La crise et ses effìts sont donc bien liés au passé des marchés, mais à un passé qui tend à s'effacer avec le temps, lentement d'ailleurs : à supposer que le coefficient du taux de croissance du crédit de 1780 à 1807 obtenu ici soit demeuré stable pour les décennies ultérieures, il faudrait attendre 1861 pour que les marchés retrouvent leur activité de 1780 .

Les régressions sur la variation des stocks de prêts tant de 1780 à 1807 que de 1807 à 1840 confirment que le crédit a fui les villes. Bien sûr, le phénomène a ses limites. Même très affaibli relativement, le marché parisien reste au sommet de la hiérarchie nationale; et, dans une région donnée, on n'observe pas non plus de bouleversement complet: en Picardie, par exemple, Corbie ne passe pas devant Amiens. Néanmoins, prêteurs et emprunteurs après la Révolution paraissent bien décidés à se passer des services d'intermédiaires des grandes 
villes ${ }^{34}$. Au contraire, durant la Révolution puis pendant la phase de reconstruction, les marchés les plus petits se comportent mieux. Pour expliquer ce constat, il faut alors recourir à des variables aptes à prédire l'intensité du déclin (de 1780 à 1807) ou la rapidité avec laquelle tel ou tel marché renoue avec la croissance (de 1807 à 1840).

On peut d'abord penser à des effets qui ont varié dans l'espace. Considérons alors une variable macro-régionale. Comme le montrent les tableaux 3 et 4 , une disparité entre le Nord et le Sud s'invente après la Révolution. Si, durant la crise, la géographie importe peu (la variable indicatrice «Nord/Sud » n'est jamais significative), pendant la période de redémarrage, les marché croissent plus vite au Nord (la variable a un coefficient positif et significatif dans les deux régressions du tableau 4). De plus, ce coefficient est élevé.

Ce découpage spatial n'est pourtant pas tout à fait une nouveauté car il renoue, par-delà le choc révolutionnaire, avec une caractéristique importante des marchés de l'Ancien Régime. Pour un marché donné, la part des rentes dans les engagements conclus en 1780 constitue un bon indicateur du risque auquel il allait se trouver exposé. Ce sont en effet les sommes prêtées sous forme de rentes qui ont été le plus systématiquement remboursées pendant l'hyperinflation. De plus, pour les obligations, les remboursements en papier déprécié ont un effet direct sur la demande (puisqu'il n'y a plus alors à refinancer les dettes qui viennent à terme) et sur l'offre du crédit (puisque la confiance est entamée). Dans le cas des rentes, où il n'y a pas nécessité de refinancement, toute la chute du crédit est à imputer à la perte de confiance des prêteurs. Or, dans des régressions (non données ici) la variable «Part des rentes en 1780 » a un coefficient toujours négatif et celui-ci est significatif pendant la crise. Cette variable est cependant liée à la taille des marchés et à la géographie de sorte qu'elle ajoute peu d'informations par rapport à ce qu'indiquent déjà les tableaux 3 et 4 .

Mais si l'organisation des marchés dans le Nord a pu les rendre plus vulnérables à la crise, elle n'a pas empêché la reconstruction d'un système de crédit au $\mathrm{XIX}^{\mathrm{e}}$ siècle. Sans doute ne peut-on exclure que les évolutions divergentes du Nord et du Sud aient simplement résulté d'un déplacement des opportunités dans l'espace : les marchés se seraient mieux rétablis au Nord parce que l'activité économique y aurait été plus soutenue. L'hypothèse semble pourtant peu probable, étant donnés l'étendue et l'hétérogénéité des espaces en cause et le fait que cette divergence entre Nord et Sud se retrouve dans des différences de pratiques que nous analysons ci-dessous et qui, elles, remontent à l'Ancien Régime. Il faut se rendre à l'évidence: les marchés locaux ont une histoire longue.

À cette étape, plusieurs conclusions se dégagent. La première est que les modèles les plus simples $(\mathrm{S} 1, \mathrm{~S} 2, \mathrm{~S} 3)$ ne rendent pas compte de la structure des marchés 
du crédit que révèle la Révolution. Disons que le quatrième scénario, plus compliqué et donnant plus de place à l'histoire, paraît plus pertinent dans la mesure où il avance que les marchés du crédit reflètent peu ou prou leur passé, même si celui-ci, loin d'être fixé de façon immuable, paraît plutôt dépendre de la réalité économique qui l'entoure. La Révolution a détruit ce qu'on a désigné comme leur capital social, mais celui-ci s'est reconstruit et il est probable que ce processus a été d'autant plus facilement mené à bien que la richesse locale était importante, ce qui a d'ailleurs dû renforcer les liens avec le passé. Reconnaissons toutefois que cette dernière assertion reste à prouver ${ }^{35}$.

Il faut ensuite souligner que le capital social dont dépendent les marchés est soumis à des effets d'échelle et peut se déprécier. Il est évident que la Révolution a agi sur ce capital social local qu'est la confiance. Encore faut-il préciser. Au lieu d'assimiler la confiance à une institution, distinguons donc, d'un côté, les règles et les techniques d'intermédiation qui déterminent la fiabilité des prêts et, de l'autre, ce qui est un pur legs des expériences passées et qui, comme tel, crée ou détruit la confiance chez les prêteurs potentiels. Pour leur malheur, les grands marchés ont été spécialement avides d'institutions; ils ont aussi été, de ce fait, rendus plus fragiles: pendant la Révolution, tous les marchés souffrirent mais ce sont les plus grands qui perdirent le plus.

Deux hypothèses peuvent alors expliquer l'évolution des marchés du crédit. Selon l'une, qui se situe dans la longue durée, les régions riches ont plus besoin d'institutions de crédit et donc s'en dotent plus et plus vite. Les marchés riches en 1780 seraient ceux qui se sont alors les mieux équipés et qui le resteront; ce serait aussi ceux qui ont le plus souffert. Selon l'autre, qui se situe dans le moyen terme, le développement des institutions accompagne la croissance. Dans les régions à forte croissance, les marchés tendraient à développer leurs institutions et le crédit augmenterait donc plus vite qu'ailleurs. Ces deux hypothèses ne sont pas exclusives l'une de l'autre. Notons pour l'instant que le rôle important de la variable mesurant la chute du crédit de 1780 à 1807 dans la croissance de 1807 à 1840 vient à l'appui de la première.

Ajoutons que les institutions soutenant le crédit que la Révolution a détruites et qui ensuite se reconstituent plus ou moins présentent un certain nombre de caractéristiques. Elles ne sont pas, comme une somme d'atomes, parfaitement divisibles, car elles ne fonctionnent pas à l'identique en ville et à la campagne. Il y a donc des économies d'échelle. Elles ne sont pas non plus un capital purement privé, car les notaires d'un lieu semblent bien partager les mêmes pratiques d'intermédiation. De plus, elles ne se reconstituent pas aussi vite partout. En fait, recréer des institutions pour le crédit semble plus difficile dans les grands marchés que dans les petits, ceux-ci se remettant plus vite de la crise que ceux-là. Enfin, il est peu probable que ces institutions aient été épaulées par la réforme des institutions 
formelles. En particulier, la création des Hypothèques aurait dû, en principe, donner naissance à un monde nouveau uniforme, où le passé local ne joue plus ni comme atout ni comme handicap. Et, en pratique, cette réforme aurait dû bénéficier au crédit urbain - surtout parisien - en permettant de diffuser dans tout le pays une information fiable sur les grosses fortunes. La création des Hypothèques aurait donc dû faciliter les transactions entre gros prêteurs et gros emprunteurs, tout en poussant celles-ci à se concentrer dans les plus grandes villes où les effets des variations individuelles de l'offre et de la demande auraient dû se trouver atténués. Mais tel ne fut pas le cas. Ce ne sont pas les institutions formelles qui ont dicté l'évolution du crédit notarié.

Interrompons ici l'examen de la crise elle-même faute d'informations pour l'explorer plus avant - la structure des marchés en 1807 (sans parler de ce qu'elle a dû être en 1797) est trop homogène pour être à même d'explication statistique. On peut, en revanche, pousser plus loin l'analyse pour la coupe de 1840, dans la mesure où il est alors - en partic - possible de distinguer ce qui relève de la confiance et ce qui relève des institutions informelles. En effet, à cette date, nous savons combien de temps un prêteur s'engage. Et s'engager à long terme c'est s'exposer, ce qui suppose que l'on ait confiance. Certes, sur ces marchés, il y a plusieurs moyens de sécuriser les contrats. L'un est de se plier à la règle généralement admise de ne prêter qu'à concurrence de la moitié de la valeur du gage. Mais il est un autre : il suffit de raccourcir la durée des contrats. Si, sur un marché, les prêteurs consentent à s'engager pour plus longtemps que sur d'autres, c'est que, plus confiants, ils ressentent moins le besoin de se rassurer de la sorte. Reprenons donc les variables utilisées plus haut pour expliquer la croissance du crédit de 1807 à 1840 et ajoutons-y la durée pondérée de chaque marché pour 1807 (tableau 5 A). Alors que ni l'activité du crédit en 1807, ni le déclin subi entre 1780 et 1807 , ni la part des rentes en 1780 , ni la taille de la ville n'apparaissent significatifs, seule la variable Nord-Sud a ici un rôle explicatif : qui plus est, elle explique à elle seule près de la moitié de la variance. Or, quand on sait combien les marchés sont différents d'un lieu à l'autre, il semble tout à fait incroyable que la confiance règne également partout dans l'ensemble de la moitié Nord du pays. Il est donc exclu que ce soit le facteur essentiel. De même, on ne peut pas croire non plus que le redéploiement du crédit dans le Nord du pays résulte simplement de la croissance. Celle-ci a sans doute contribué à la vigueur de certains marchés. On le verra plus bas, sur l'exemple de places comme Amiens ou Troyes. En 1840 (tableau 5 B), la variable Nord-Sud reste importante même si, après la phase de rattrapage entre 1807 et 1840 , la hiérarchie des marchés s'est rétablie: les marchés les plus gros ont en effet les durées les plus longues. Cette variable Nord-Sud est d'interprétation difficile car l'industrialisation est une réalité locale, au plus régionale. En tout cas, le phénomène ne couvre pas la moitié du pays. De plus comment expliquer sur cette base l'activité des marchés du Nord qui restent à l'écart de l'industrialisation ? Ou encore comment comprendre pourquoi, entre 1780 et 1840 , le crédit croît deux fois plus vite à Troyes qu'à Amiens? 


\section{Tableau 5 - Poids de certaines variables susceptibles d'expliquer la durée des prêts en 1807 et 1840}

Partie B : Durée des prêts en 1840

\begin{tabular}{|c|c|c|c|c|c|}
\hline & Coeff. & $\begin{array}{c}\text { t de } \\
\text { Student }\end{array}$ & & Coeff. & $\begin{array}{c}\mathrm{t} \text { de } \\
\text { Student }\end{array}$ \\
\hline Constante & 1,11 & 1,67 & Constante & 0,71 & 1,24 \\
\hline Nord/Sud & 1,04 & 2,27 & Nord/Sud & 1,29 & 3,8 \\
\hline Part des rentes en 1780 & 2,83 & 3,42 & Part des rentes en 1780 & 0,71 & 1,1 \\
\hline \multirow{2}{*}{ Log volume de prêts en 1780} & $-0,07$ & $-0,55$ & Log volume de prêts en 1807 & 0,17 & 1,7 \\
\hline & & & Durée des prêts en 1807 & 0,25 & 12,8 \\
\hline $\mathrm{R}^{2}$ & 0,32 & & $\mathrm{R}^{2}$ & 0,49 & \\
\hline $\mathrm{R}^{2}$ ajusté & 0,29 & & $\mathrm{R}^{2}$ ajusté & 0,45 & \\
\hline Écart type & 1,59 & & Écart type & 1,15 & \\
\hline $\mathrm{Nb}$ d'observations & 65 & & Nb d'observations & 65 & \\
\hline
\end{tabular}

Note: La durée des prêts est en années.

La réponse tient sans doute en partie au rôle des institutions informelles. Considérons-les ici comme émanant des intermédiaires, c'est-à-dire soit des notaires soit de leurs principaux concurrents, les banquiers. Deux questions se posent: où les institutions informelles se développent-elles facilement et où viennent-elles appuyer ou concurrencer la confiance et les institutions formelles? Partir de la situation des notaires plutôt que de celle des banquiers présente ici un grand avantage car, étant soumis à un contrôle public, les premiers ont laissé des archives abondantes et systématiques. Nous pouvons donc compléter notre échantillon des actes de prêts de 1840 par les renseignements établis à l'époque sur les transmissions d'études notariales, soit quelque sept cents dossiers portant sur les années 1840-1843 ${ }^{36}$. Ces dossiers nous informent sur l'activité de l'étude cédée, son prix, le nombre des concurrents à la reprise ainsi que sur les aptitudes et la formation des entrants.

On perçoit mieux ainsi le fonctionnement des institutions informelles de crédit puisqu'on peut par là préciser la façon dont se constitue le capital humain des notaires. Pour entrer dans la profession, il fallait un apprentissage comme clerc pendant cinq à sept ans de stages dont une partie dans une ville située plus haut dans la hiérarchie judiciaire que celle où le postulant entendait finalement s'établir. N'était cette obligation, rien ne dictait les limites géographiques de l'apprentissage du futur notaire qui était donc libre de choisir de faire un tour de France ou un tour de canton. Or deux conclusions se dégagent de l'examen des périples effectués. D'une part, l'apprentissage du Nord n'est pas celui du Sud, même si la ligne de démarcation est franchie par un petit nombre de méridionaux qui vont se former à Paris et si la frontière devient parfois floue, comme dans les départements de la Charente et de la Charente-Maritime où les notaires hésitent entre une formation 
méridionale à Bordeaux (utile pour les liens avec le commerce du vin) et un tropisme nordique qui peut les entraîner jusqu'à Rennes, Orléans, Paris ou même Rouen. D'autre part, les apprentis notaires du Nord et du Sud organisent très différemment le déroulement de leurs stages successifs. Au Sud, les pérégrinations des clercs suivent une distribution franchement bimodale : à un pôle, il y a ceux qui ne bougent pas, à l'autre ceux qui font le grand saut vers Paris quitte à parcourir souvent plus de $500 \mathrm{~km}$. Dans la moitié Nord, au contraire, les distances parcourues se distribuent de façon beaucoup plus concentrée avec de moins en moins de cas lorsqu'on s'éloigne de la moyenne (tableau 6).

\section{Tableau 6 - Les circuits d'apprentissage des notaires}

Panneau A : Distances parcourues pendant les stages (en kilomètres)

\begin{tabular}{|c|c|c|c|c|}
\hline \multirow{2}{*}{$\begin{array}{l}\text { Région de naissance } \\
\text { Type de transmission }\end{array}$} & \multicolumn{2}{|c|}{ France du Nord } & \multicolumn{2}{|c|}{ France du Sud } \\
\hline & Famille & Externe & Famille & Externe \\
\hline Distance parcourue: très petite ( $1^{\text {er }}$ quartile $)$ & 17 & 32 & 3 & 21 \\
\hline Distance parcourue: petite ( $2^{-}$quartile) & 79 & 96 & 56 & 83 \\
\hline Distance parcourue : grande ( $3^{\mathrm{e}}$ quartile) & 229 & 197 & 138 & 217 \\
\hline Distance parcourue : très grande ( $4^{e}$ quartile $)$ & 463 & 465 & 462 & 612 \\
\hline Distance maximum & $\begin{array}{c}827 \\
3.50 \%\end{array}$ & 1740 & 404 & 1118 \\
\hline Stages purement locaux & $3,50 \%$ & $1,90 \%$ & $21 \%$ & $19 \%$ \\
\hline $\mathrm{N}$ & 36 & 363 & 91 & 198 \\
\hline
\end{tabular}

Panneau B : Régions visitées (nombre de stages)

\begin{tabular}{|c|c|c|c|c|c|}
\hline & & \multicolumn{2}{|c|}{ Région de stage } & \multicolumn{2}{|c|}{ Cour d'appel } \\
\hline & & Nord & Sud & de naissance & de Paris \\
\hline Région de naissance & Nord & 1094 & 51 & 778 & 256 \\
\hline Id. & Sud & 65 & 529 & 472 & 62 \\
\hline
\end{tabular}

Panneau C: Régions d'exercice (nombre de notaires)

\begin{tabular}{lcccccc}
\hline & \multicolumn{2}{c}{ Région d'exercice } & & \multicolumn{2}{c}{ Cour d'appel } \\
\cline { 7 - 8 } & & Nord & Sud & & de naissance & autres \\
\hline Région de naissance & Nord & 386 & 27 & 309 & 104 \\
$I d$. & Sud & 9 & 269 & 257 & 21 \\
\hline
\end{tabular}

Source: AN BB10, Dossiers de notaires, 1838-1843.

Note: On oppose les cessions d'étude entre parents (où l'asymétrie d'information est faible) et entre non-parents (où il peut y avoir une asymétrie d'information).

Des régimes régionaux se dessinent donc. Au Sud, deux coexistent. Dans l'un, les futurs notaires vont acquérir leur formation au loin, et parfois jusqu'à Paris, lorsqu'ils se destinent à exercer leur charge dans une préfecture ou au siège d'une cour d'appel. Au contraire, ceux qui, pour une raison ou pour une autre (manque d'argent, de capacités...) s'en tiennent à une formation locale limitent leur horizon aux frontières d'un canton ou de quelques communes. Pour ces derniers, apprendre 
au loin n'est guère utile et mieux vaut faire des stages dans le voisinage, voire rester chez soi. Par comparaison, au Nord, la mobilité est plus forte et le passage par une grande ville plus fréquent, même s'il ne dure pas forcément longtemps. Et pour ceux qui convoitent une étude urbaine, il est courant de faire un passage par Paris, même si ce n'est pas la règle générale. Dans tous les cas, ceux du sud de la Loire ont peu à apprendre dans le Nord, et vice versa.

Ces régimes régionaux correspondent à des équilibres différents qui entretiennent le crédit par le truchement d'institutions spécifiques. Chacun d'eux peut se caractériser par un petit nombre d'indicateurs, à commencer par la formation que se donnent les notaires et le degré de contrôle qu'ils exercent sur les marchés locaux du crédit. Nous distinguerons de ce point de vue la France du Nord de celle du Midi et isolerons dans chaque cas les marchés des grandes villes (celles de plus de 10000 habitants) et les autres. Par commodité, nous parlerons de marchés urbains et de marchés ruraux.

Commençons par les notaires ruraux du Sud. Dans ces petits marchés où le crédit était déjà plus de moyen terme que de long terme avant 1780 et où il n'y avait, bien sûr, pas de banques, la crise de la Révolution a été de relativement faible ampleur et s'est vite résorbée. Cette reprise en douceur est le fait des notaires locaux qui ont rapidement restauré leur activité d'intermédiaires de crédit. Avant comme après la Révolution, celle-ci repose sur un savoir tout informel comme le montre la formation que se donnent les entrants. Ce savoir se transmet en effet soit à l'intérieur de la famille, soit à des clercs qui ont fait l'essentiel ou la totalité de leur apprentissage dans leur future étude. Ni Abraham ni Ulysse, les notaires sont donc surtout sédentaires. De plus, ils occupent le terrain autant qu'il est permis et rares sont les cantons où l'effectif maximum de notaires autorisé ne soit pas atteint (cinq). Enfin, ils adoptent résolument ce qui constitue une des grandes innovations financières de la première moitié du XIX ${ }^{\mathrm{e}}$ siècle: la lettre de change notariée. Ce type d'intermédiation repose avant tout sur la réputation des notaires et sur leur capacité de mobiliser un réseau local.

Dans cette configuration, transférer sa réputation devient primordial et s'opère avant tout à l'intérieur des familles. Ce désir de rester entre soi est tel que lorsqu'un notaire décède après avoir repris l'étude de son père, c'est souvent celuici qui reprend du service jusqu'à ce que son petit-fils soit en âge de lui succéder à son tour, à moins qu'il ne trouve un remplaçant qui s'oblige à laisser la place un ou deux ans plus tard. Les autorités de tutelle sont certes critiques devant ce genre de gymnastique dynastique, mais elles préfèrent fermer les yeux sur ces pratiques douteuses plutôt que voir des études péricliter. Par ailleurs, l'équilibre ainsi réalisé implique que chaque étude rende ses clients captifs. Puisque la clientèle ne fait confiance qu'à son notaire de père en fils, elle ne peut avoir recours qu'à lui qui doit, en retour, éviter que son savoir ne s'ébruite. Mais les affaires risquent de souffrir de trop de cloisonnement. Or, s'il s'ouvre tant soit peu, le système doit passer par des correspondants, ce qui suppose un certain partage d'informations. Un tel élargissement est certes pensable s'il sait rester limité. De là l'intérêt pour les prêts à court terme. Le notaire qui rédige des lettres de change leur offre une 
garantie implicite. Pour autant, il garde par devers lui les informations qu'il détient sur la solvabilité à long terme de ses clients. Ce faisant, il peut s'autoriser un certain œcuménisme dans le choix de correspondants chez qui les lettres de change doivent être tirées. Même si ces derniers résident en général à moins de vingt kilomètres de là où ses clients habitent, le notaire ne craint pas de mettre en relation ses clients avec un autre notaire, un marchand, voire avec un banquier puisqu'il est assuré de conserver le monopole de son savoir sur eux.

Dans le Sud rural, le crédit repose essentiellement sur les notaires qui assurent un semi-long terme par les obligations et un court terme par les lettres de change. Les banquiers sont presque absents ou plutôt leur diffusion est ici très lente. À court terme, un tel système est assurément efficace. Il donne aux notaires de fortes incitations pour réinvestir dans leur savoir après la Révolution, puisqu'ils restent en situation de monopole. Le système ne demande aucun investissement public, et le capital social est l'ensemble des représentations qui maintient chacun dans une stratégie de relation binaire ( 1 client, 1 notaire). Tout le reste est le capital privé du notaire. En revanche, son inconvénient est de ne pas offrir une intermédiation variée et de restreindre les possibilités de croissance du crédit.

Dans le Nord rural, le crédit repart sur des bases différentes. Les notaires ne s'investissent que rarement dans le crédit à court terme ${ }^{37}$. Ils ont aussi moins d'attaches sur place et leur formation passe par des stages moins locaux que leurs collègues méridionaux (tableau 7). Ils paient aussi plus cher les études où ils s'installent. Toutes ces différences suggèrent que l'équilibre du Nord rural est plus ouvert qu'au Sud et qu'il est plus facile de transmettre des informations d'un notaire à un autre (et surtout d'un notaire d'un lieu à un notaire d'un autre lieu). Ce n'est pas que le pur mécanisme de réputation soit ici sans importance, mais les renseignements sur la valeur des études ou sur la qualité des emprunteurs et des prêteurs peuvent aussi circuler autrement (et mieux) à l'intérieur d'une région. Un exemple: celui qui devient premier clerc de l'étude qu'il va reprendre ne passe souvent que quelques mois à ce poste, ce qui suggère qu'il n'en faut pas plus pour s'informer sur la valeur de l'entreprise. C'est là un bon indice. Tout indique aussi que les entrants se satisfont des clauses du contrat de vente et qu'ils les jugent suffisantes pour s'assurer des clientèles. Ce système de renouvellement externe a pour conséquence de rendre inessentielle au bon fonctionnement du crédit l'implication personnelle du notaire. On évite ainsi qu'une minorité de notaires chenus, riches en années et en expérience, exclue du crédit une plèbe de jeunes forcément plus ignorants des caractéristiques de leurs ouailles. Dans ces conditions, le lien entre la garantie formelle (immobilière) que propose l'emprunteur et le prêt qu'il

\footnotetext{
37 - De nombreux notaires ont une activité de crédit à court terme soit en tant que prêteur, soit comme emprunteur. Quand ces transactions ont laissé des traces, elles portent sur des sommes importantes mais ne touchent qu'un public restreint. Tantôt les notaires acceptent des dépôts d'argent (qu'ils placent ensuite à long terme), tantôt ils consentent des avances à court terme à quelques clients bien choisis. C'est dire que ni dans un cas ni dans l'autre il ne s'agit d'une version nordique de la lettre de change notariée.
} 
reçoit peut être plus direct au Nord qu'au Sud. Sans aller jusqu'à impliquer le passage par un bureau des Hypothèques, un prêt demande avant tout des gages immobiliers. De là, le règne sans partage de l'obligation et l'absence des lettres de change notariées. Mais comme le contrat à gage immobilier est particulièrement coûteux en frais fixe, il s'ensuit que les prêts doivent ici être plus longs ${ }^{38}$. Partant, s'enfermer dans sa clientèle serait un grave handicap car au sein d'une même étude offre et demande de crédit s'accorderaient difficilement. Il importe donc d'avoir des correspondants, mais pour des prêts à long et non plus à court terme comme dans le Sud. Les déambulations des notaires ruraux pendant leur apprentissage s'expliquent ainsi par le besoin de constituer des réseaux de notaires et de clients pour équilibrer ces marchés et non par une propension plus grande que dans le Sud à acquérir de la formation. Pour ce qui est du crédit, le Sud est plus sophistiqué que le Nord, alors que les autres contrats notariés semblent à peu près identiques. Enfin, dernière conséquence de cet équilibre, au lieu de faire du court terme (ce qui créerait trop d'informations asymétriques), les notaires du Nord vont donc partager l'activité du crédit avec des banques locales beaucoup plus tôt que dans le Sud. Ils sont, en revanche, peu soucieux de recourir aux nouvelles institutions formelles que sont les Hypothèques, la masse des clients ruraux (petits et moyens) n'en faisant pour ainsi dire aucun usage.

\section{Tableau 7 - Pourcentages des nouveaux notaires succédant à un parent (situation vers 1840 )}

\begin{tabular}{|c|c|c|c|c|c|}
\hline & \multicolumn{3}{|c|}{ Quand leur dernier stage a été effectué } & \multirow[t]{2}{*}{ Ensemble } & \multirow{2}{*}{$\begin{array}{c}\text { Nombre } \\
\text { d'observations }\end{array}$} \\
\hline & $\begin{array}{l}\text { dans l'étude } \\
\text { où ils s'installen }\end{array}$ & $\begin{array}{l}\text { dans la même } \\
t \quad \text { commune }\end{array}$ & ailleurs & & \\
\hline \multicolumn{6}{|c|}{ France du Nord } \\
\hline Grandes villes & 16,7 & 6,7 & 4,8 & 7,1 & 48 \\
\hline Autres & 30,6 & 18,2 & 3,5 & 13 & 253 \\
\hline \multicolumn{6}{|c|}{ France du Sud } \\
\hline Grandes villes & 37 & 15,4 & 6,3 & 20,6 & 56 \\
\hline Autres & 53,4 & 61,1 & 14,2 & 28,9 & 261 \\
\hline
\end{tabular}

Source: AN BB10, Dossiers de notaires, 1838-1843.

Note: On a négligé les notaires ayant déjà exercé; grandes villes équivaut ici à préfectures et sous-préfectures.

Quoi qu'il en soit, les marchés où ce système se met en place sont ceux où l'activité de crédit progresse le plus entre 1807 et 1840, qu'ils aient été éprouvés ou non par la Révolution. Cette croissance repose sur des pratiques qui, loin d'être locales, s'étendent sur toute la moitié Nord du pays. On peut, à nouveau, faire l'hypothèse que la plus grande richesse et donc la plus grande valeur des gages

38 - Contrairement à ce qu'on observe dans le Sud, le notaire ici sera dans l'impossibilité de financer lui même des prêts - forcément assez longs - compte tenu du fait qu'il ne dispose malgré tout que d'un capital restreint. 
ont pu orienter cette partie du pays vers cet équilibre plutôt que vers celui qu'on a observé au Sud.

Des équilibres distincts pour les grandes villes font-ils pendant à ces deux équilibres qu'on observe pour les campagnes? En d'autres termes, faut-il supposer que le monde urbain du Nord comme celui du Sud ont, chacun pour leur part, construit des institutions propres sur la base de leur histoire particulière? Plusieurs éléments incitent à le penser. Ainsi les marchés urbains du Sud se singularisentils en réalisant la performance la plus mauvaise entre 1807 et 1840 : ils font moins bien que les marchés ruraux proches et que l'ensemble des marchés du Nord, ruraux et urbains confondus. De plus, comme dans le Sud les notaires ruraux sont nombreux, la coopération avec leurs homologues en ville est rendue difficile. Enfin, le développement de la lettre de change notariée implique que les études rurales et urbaines du Midi ont tissé entre elles des relations qui n'ont pas d'équivalent dans le Nord. Si tous les notaires urbains du Sud ne pratiquent pas la lettre de change, la plupart sont des correspondants de notaires du voisinage rural. Enfin les circuits de formation pour notaires urbains du Sud et du Nord ne se croisent qu'à Paris : si les notaires du Sud sont peu tentés de passer la Loire, ceux du Nord ne paraissent même pas l'avoir envisagé. Leurs formations semblent donc différentes.

À l'inverse, d'autres éléments suggèrent qu'en 1840 un système de crédit urbain unique tend à s'établir. Pour les notaires urbains méridionaux, sans doute est-il important d'être destinataires dẹ lettres de change ; mais ce n'est malgré tout qu'une fonction parmi d'autres et, comme leurs collègues du reste de la France, ils privilégient l'obligation. Cette spécialisation dans les moyen et long termes a une implication: on trouve, à population égale, autant de banquiers dans les villes du Sud que dans celles du Nord. Dans cette mesure, une certaine intégration des intermédiaires financiers entre les villes du Nord et du Sud semble probable; elle est, en tout cas, nettement plus poussée que pour les campagnes; nous reviendrons ailleurs sur la question de savoir quand et comment ces pratiques urbaines sont devenues homogènes sur tout le territoire.

Il reste que les notaires urbains ne font pas aussi bien que ceux des campagnes. S'agit-il d'un rattrapage, les campagnes parvenant alors à surmonter le retard qu'elles auraient pris par rapport aux marchés urbains du crédit? Faut-il plutôt admettre que ceux qui, sous l'Ancien Régime, empruntaient ou prêtaient en ville sont allés faire affaire chez des notaires ruraux ${ }^{39}$ ? La seconde hypothèse au moins est improbable. On l'a dit, la redistribution du crédit de 1780 à 1840 s'est faite par la disparition d'un certain nombre de gros prêts urbains et l'explosion de petits prêts ruraux. Or les destinataires des uns ne sont évidemment pas les destinataires des autres. La demande de prêts se serait-elle donc déplacée des villes vers la campagne? La supposition est plausible de 1780 à 1807, voire jusqu'en 1820, non après. En effet, la France d'alors s'urbanise et s'industrialise, lentement

39 - Si l'on accepte que, dans le Nord, les réseaux de crédit englobent les villes et que les villes où les notaires ruraux se forment sont au sommet de réseaux hiérarchisés qui redistribuent le capital ici ou là selon les besoins, alors il faudrait mesurer la croissance du crédit par réseaux, ce que nos échantillons ne nous permettent pas de faire. 
peut-être mais sûrement, et l'on devrait donc observer une demande croissante dans les villes - et plus rapidement croissante que dans les campagnes. Or c'est l'inverse qui se produit, résultat surprenant. Tient-il à un report des créanciers et des débiteurs urbains sur le crédit à court terme, voire à un recul global de l'ensemble des formes de crédit, à court et à long terme, notarié et non notarié ?

On peut aussi expliquer cette faible croissance du crédit notarié urbain par les hésitations de la profession sur la meilleure façon de reconstruire le crédit. Quelle stratégie retenir, en effet? Fallait-il privilégier les rapports informels avec la campagne ou ceux, plus formels, qui se nouaient de ville à ville? Et quel type de prêt promouvoir? Valait-il mieux se concentrer sur les affaires immobilières et sur les prêts hypothécaires en tissant des réseaux de crédit à long terme de ville à ville ou plutôt tolérer un certain glissement vers le court terme, voire l'encourager? Dans tous les cas, les notaires urbains ont alors du mal à se mettre d'accord sur les pratiques à adopter et les investissements à consentir. Leur nombre même constitue pour eux un handicap supplémentaire par rapport à leurs homologues ruraux, puisqu'ils ont à faire face à de graves problèmes de coordination. De telles difficultés doivent certes se trouver atténuées là où l'industrie se développe; mais, on le sait, le phénomène est loin d'être général, notamment dans le Sud.

Le cas le plus surprenant est celui de la capitale. Après avoir été florissant au XVIII ${ }^{\mathrm{e}}$ siècle, le marché du crédit parisien connaît en effet une longue stagnation. Réduit à presque rien à la fin de la Révolution, il se comporte ensuite particulièrement mal ; beaucoup plus mal qu'on ne s'y attendrait. Même sans que se reconstitue le système de coopération entre les notaires qui s'était instauré à la fin de l'Ancien Régime, l'utilisation de l'information produite par l'administration des Hypothèques aurait dû drainer les transactions des riches vers la capitale. Or cela ne se produit pas, du moins avant la croissance du Crédit foncier sous le Second Empire qui provoque à la fois une (tardive) envolée du crédit notarié et une concentration des gros prêts dans la capitale. Faut-il alors en revenir aux présupposés classiques selon lesquels des intermédiaires d'Ancien Régime comme les notaires étaient et ne pouvaient être que peu capables d'assurer la croissance de l'endettement à garantie immobilière? On retrouverait là l'idée que rien d'important ne se serait passé avant que les banquiers prennent le relais des notaires.

Pour en juger, on voudrait estimer l'interaction entre banques et la croissance du crédit notarié. Mais l'exercice est difficile. D'abord parce que les sources qui détaillent le nombre des banques dans les villes ne permettent guère de remonter au-delà de la fin des années 1820 et sont d'interprétation délicate. L'Almanach du commerce repère trois banques en 1829 à La Châtre : cela signifie-t-il que ces trois banques viennent de s'ouvrir ou que l'Almanach s'est enfin décidé à les répertorier? Ensuite parce que les liens entre crédit notarié, nombre de banques et taille des villes sont très forts (la corrélation entre le nombre de banques dans un marché donné, la taille de la ville principale et le stock des prêts notariés est de plus de 0,8 ), et qu'on ne peut pas identifier correctement le sens de l'interaction. À tout le moins, les banques et le crédit notarié se développent dans les même lieux. En outre, il apparaît que le nombre des banquiers dans un lieu y augmente la valeur 
des offices (tableau 8). Comme la valeur des offices dépend de leur activité et que celle-ci est en grande partie une activité d'intermédiaire de crédit, c'est bien là un effet positif entre crédit notarié et crédit bancaire qui est mis en évidence. II semble donc que, en province au moins, banques et crédit notarié s'appuient mutuellement. On serait là aux antipodes du cas parisien. À ce titre, il est intéressant d'illustrer cette complémentarité entre crédit bancaire et crédit notarial par le marché de Troyes qui, de tous ceux de notre échantillon, est celui dont l'évolution s'éloigne le plus de celle du marché parisien. Cette place dont l'industrie textile connaît alors une croissance rapide voit son activité de crédit traverser sans mal la crise révolutionnaire puis, celle-ci passée, ses intermédiaires, loin d'être paralysés par l'incertitude, développent leurs affaires à un rythme très soutenu. À Troyes, le crédit connaît donc une flambée ininterrompue : le stock des prêts double entre 1780 et 1807 , et à nouveau de 1807 à 1840 . De plus banques, courtiers de commerces et assurances sont tous présents dès 1840 .

\section{Tableau 8 - Poids de certaines variables susceptibles d'expliquer la valeur des offices de notaire en France (situation vers 1840)}

\begin{tabular}{|c|c|c|c|c|c|c|}
\hline & \multicolumn{2}{|c|}{ Ensemble } & \multicolumn{2}{|c|}{ Sans Paris } & \multicolumn{2}{|c|}{ Communes $>3000 \mathrm{hab}$} \\
\hline & Coeff. & $\begin{array}{c}\mathrm{t} \text { de } \\
\text { Student }\end{array}$ & Coeff. & $\begin{array}{c}\text { t de } \\
\text { Student }\end{array}$ & Coeff. & $\begin{array}{c}\mathrm{t} \text { de } \\
\text { Student }\end{array}$ \\
\hline Constante & 12,25 & 2,35 & 13,56 & 2,62 & 32,4 & 2,32 \\
\hline \multicolumn{7}{|l|}{ Type de commune: } \\
\hline Préfectures & 45,5 & 4,89 & 42,93 & 4,68 & 42,91 & 3,19 \\
\hline Sous-préfectures & 15,13 & 2,64 & 14,97 & 2,67 & 2,39 & 0,25 \\
\hline Autres & 6,01 & 1,61 & 5,76 & 1,58 & $-17,35$ & $-1,32$ \\
\hline Nord/Sud & 31,93 & 9,22 & 31,67 & 9,32 & 32,61 & 4,05 \\
\hline Population & 0,000027 & 0,11 & 0,0001 & 0,48 & 0,0006 & 1,46 \\
\hline Nombre de censitaires/hab. & 993,26 & 3,13 & 1006,71 & 3,24 & 3041,77 & 3,11 \\
\hline Nombre de banquiers & 1,66 & 2,27 & 1,86 & 2,54 & 0,75 & 0,68 \\
\hline Nombre de notaires & $-0,42$ & $-0,43$ & $-0,77$ & $-0,79$ & $-2,45$ & $-1,49$ \\
\hline $\mathrm{R}^{2}$ & 0,76 & & 0,5 & & 0,52 & \\
\hline $\mathbf{R}^{2}$ ajusté & 0,76 & & 0,49 & & 0,49 & \\
\hline Écart type & 37,18 & & 36,42 & & 48,77 & \\
\hline Observations & 543 & & 533 & & 185 & \\
\hline
\end{tabular}

Sources: AN BB10, Dossiers de notaires, 1838-1843; Almanach du commerce, Bottin de 1840 (pour le nombre de banquiers et de censitaires).

Notes: La variable dépendante est le prix d'un office de notaire en milliers de francs.

"Nord/Sud" est une variable indicatrice pour les communes des départements situés au Nord d'une ligne allant de La Rochelle à Genève en passant par Poitiers, Moulins et Lyon. La variable "Nombre de censitaires par habitant» dans la commune où est située l'étude est considérée comme un indicateur de richesse.

La trajectoire de ce marché qui semble indifférente aux à-coups pourtant si brutaux de l'histoire suggère que, pour expliquer le devenir des institutions financières, outre les variables déjà étudiées, il importe de prendre en compte la richesse en acte, ou en puissance: celle qui existe à un moment donné mais aussi celle que semble promettre le futur. Si un marché est pratiquement toujours plus 
actif au Nord qu'au Sud, si les prêts y sont plus gros et durent plus longtemps, sans doute est-ce parce que le Nord est riche. Cette richesse a l'avantage d'augmenter l'offre de prêts et de densifier l'intermédiation du crédit. Seuls les marchés riches peuvent s'offrir «fromage et dessert», notaires et banquiers, et ce luxe ne nuit pas aux notaires puisque la valeur de leur charge augmente là où il y a plus de banquiers. Dans les régions riches, les institutions formelles sont complémentaires d'institutions informelles. Pour revenir sur l'exemple de Troyes, sur cette place vers 1840 , les notaires font des obligations avec hypothèques - institutions formelles - en même temps qu'ils animent avec les banquiers un marché du court terme où les institutions importantes sont informelles.

Nous avons tenté de comprendre comment se détruisent et se reconstruisent les marchés du crédit pendant et après la Révolution française. Ce faisant, nous avons identifié divers mécanismes qui se mirent en place entre 1807 et 1840 pour recréer la confiance et permettre l'oubli de la catastrophe de la Révolution.

Soulignons d'abord que ces mécanismes sont très variés puisqu'ils vont de la réputation personnelle à l'utilisation d'une institution formelle à vocation universaliste comme les Hypothèques. De même, ils englobent les pratiques des courtiers de crédit comme celles des intermédiaires financiers. Mais cette variété se développe dans l'espace d'une façon systématique, ce qui impose certaines contraintes à la recherche.

La première contrainte concerne l'espace à considérer. Si, comme c'est souvent le cas, on étudie la Révolution et ses effets à partir d'observatoires particuliers - une ville par exemple, ou une région -, sachant sa vocation nationale, on a tendance à croire que les institutions qui prévalent dans un lieu sont les mêmes partout, sauf à admettre qu'elles sont le produit d'éléments locaux (un capital social). En embrassant un espace large, ce genre de conclusion n'est plus permis. Pour comprendre les marchés financiers dans un lieu ou une région, il faut accepter de les confronter à d'autres lieux ou d'autres régions qui, n'ayant pas les mêmes expériences, permettent de contrôler l'argumentation.

Deuxième contrainte: la durée. Dès lors qu'on s'intéresse aux institutions ou au capital social, il faut se donner un observatoire couvrant une période assez longue pour que ces objets puissent évoluer. À court terme, on peut constater l'existence d'un cercle vertueux liant richesse, crédit, croissance et institutions qui entretiennent la confiance. Toute étude en coupe ne fait qu'évaluer l'intensité de ce cercle vertueux. Un observatoire sur le moyen terme permet plus. On peut ainsi espérer comprendre comment se maintiennent la distribution de ces institutions de confiance et de crédit. On ignorera en revanche comment elles se sont construites, sauf à allonger - beaucoup - le temps d'observation; et à veiller à ce que la période examinée comporte des chocs importants. De là, la nécessité pour le projet de traverser deux époques (avant et après la Révolution): en l'occurrence, c'est le choc peu attendu de la Révolution qui révèle les structures.

Ces contraintes acceptées, l'analyse permet un ensemble de conclusions. Plus les marchés sont actifs, plus leurs institutions formelles sont complémentaires de 
leurs institutions informelles. La règle vaut pour les banques et les notaires, et pour leurs interactions. Elle vaut aussi pour les institutions qui entretiennent la confiance (formelles ou informelles). Ainsi, dans la moitié Nord du pays, les notaires ruraux se sont-ils appuyés tant sur des institutions formelles comme les Hypothèques que sur des circuits informels d'apprentissage et de circulation de clients. De même, au Nord encore, la diffusion des banques - organisations sans institutions formelles complémente le crédit notarial qui est, lui, encadré par une série d'institutions formelles. Le choix n'est donc pas entre formel et informel. Dans chaque marché, il s'agit bien plutôt de décider quel mélange retenir et, surtout, de quelles institutions spécifiques s'équiper. Par ailleurs, les institutions sont complémentaires de la richesse locale. Plus les marchés sont riches, plus ils sont à même de payer le coût des institutions, et plus les institutions sont denses, plus elles sont capables d'entretenir et d'augmenter la richesse. Plaçons-nous quelque part dans les hautes terres du Massif central, disons dans un gros bourg comme Salers. Si, dans un tel lieu, il n'y a pas de banques et si les notaires se contentent d'y animer le crédit dans la solitude de leur réputation personnelle, ce n'est pas parce que le régime financier est enfermé dans un carcan de règles étatiques. Le problème est bien plutôt que dans cette région il n'existe ni une richesse passée ni un avenir radieux susceptibles de créer une masse d'actifs financiers suffisante pour entretenir un système de crédit vraiment consistant.

Ces conclusions se retrouvent dans les systèmes distincts observés au Nord et au Sud, en ville et à la campagne: En allant du Sud rural au Nord urbain, on passe ainsi d'un monde à institutions informelles avec un seul type d'intermédiaire et une activité de crédit faible, à un monde à institutions informelles et formelles, avec une diversité d'intermédiaires spécialisés et une activité de crédit forte. Cet ordre est ici identifié en 1840 mais sans doute prévalait-il déjà sous l'Ancien Régime, au moins pour une part. Et, de ce point de vue, la Révolution a agi comme un révélateur, en détruisant les structures d'intermédiation et ruinant la confiance dans les institutions existantes. De ce fait, elle a touché le Nord plus que le Sud et les villes plus que les campagnes.

Suivre l'évolution des marchés jusqu'en 1840 permet de voir se reconstituer la structure hiérarchisée du crédit et donc, en particulier, de mieux saisir le rôle joué pendant la phase de reconstruction par la confiance. Celle-ci se révèle, en effet, ne pas être un acquis durable, mais bien plutôt le résultat des institutions qui rendent les emprunteurs fiables. Si le Nord du pays a plus souffert que le Sud, il se rééquipe néanmoins plus vite en institutions, de sorte qu'en 1840 il abrite des prêteurs qui semblent plus confiants. Disons que la confiance est le résultat d'investissements institutionnels plus ou moins réussis.

Ajoutons que le capital social résulte très largement d'investissements à long terme qui semblent liés à l'activité économique et à la richesse de chaque marché. De fait, on l'a vu, les pratiques d'intermédiation sont spécifiques aux régions et, à l'intérieur des régions, aux marchés locaux. Quand la Révolution détruit le système de prêt de l'Ancien Régime, les marchés souffrent mais les structures d'intermédiation se reconstruisent toujours, selon deux modalités. Soit en recourant à des informations informelles et personnelles - solution qui se révèle sans avenir car elle 
enferme les clients des notaires dans des relations très étroites. Soit, en passant par des institutions plus formelles comme les Hypothèques et le partage d'informations entre intermédiaires. Dans cette seconde solution, qui est plus ardue au départ, les institutions formelles et informelles sont complémentaires. Mais leur mise en place dépend alors ou de la richesse du marché, ou des opportunités qui s'y rencontrent. Ainsi, à Dijon qui est assurément une ville riche avant comme après la Révolution mais dont l'activité économique ne connaît pas de grand succès après 1789 , le marché du crédit progresse à un rythme presque aussi soutenu qu'à Troyes plus pauvre mais dont l'activité profite d'un véritable boom textile.

Plusieurs phénomènes jouent dans la renaissance des marchés du crédit après la Révolution. Puisque les marchés les plus actifs ont le plus souffert et se remettent lentement, il s'ensuit que le capital social ne constitue pas un acquis durable mais, s'il peut se perdre, il peut aussi se reconstituer. De plus, qu'une crise puisse détruire la patiente accumulation d'informations nécessaires au fonctionnement des marchés du crédit, c'est ce dont témoigne clairement le recul et la décentralisation du crédit entre 1780 et 1807 . Enfin, la reconstruction des marchés demande de nouvelles techniques d'information pour appuyer les prêts. Mais si cette exigence est aisée à satisfaire dans de petits marchés où la réputation du notaire peut facilement remplacer d'autres mécanismes qui avaient jusque-là assuré la confiance, elle l'est beaucoup moins dans les marchés importants. La réputation a ses limites et les autres institutions ne se reconstruisent que lentement.

En prenant comme unité d'analyse des marchés, nous espérions identifier des pratiques de crédit différenciées et dégager le rôle qu'y tenait le capital social local. Sur ce point l'historiographie proposait deux pistes opposées. L'une considérait que le capital institutionnel se constituait au niveau le plus local: celui d'une ville ou, au plus, d'une région. L'autre le concevait comme uniforme, en prenant pour acquis le succès du projet Jacobin. Au terme de cette recherche, c'est plutôt une France de longue durée qui se dessine, où s'oppose un Nord dont les marchés de crédit sont centrés sur du long terme et un Sud où domine le crédit à court et moyen terme. Cette opposition se retrouve dans les contrats, dans le choix des intermédiaires et dans les circuits de formation de ces derniers - et elle a un très grand pouvoir explicatif. Elle ne renvoie donc ni à une multitude de régions ni à une entité nationale, plutôt à des espaces propres à des marchés qui se construisent au gré de l'histoire.

Philip T. Hoffman Caltech

Gilles Postel-Vinay EHESS/INRA-LEA

Jean-Laurent Rosenthal UCLA/INRA-LEA 